\title{
Les objets de Jimmie Durham en conversation : au défi de l'art contemporain
}

\section{Sophie Moiroux}

\section{(2) OpenEdition}

1 Journals

Édition électronique

URL : http://journals.openedition.org/imagesrevues/153

DOI : 10.4000/imagesrevues.153

ISSN : 1778-3801

Éditeur:

Centre d'Histoire et Théorie des Arts, Groupe d'Anthropologie Historique de l'Occident Médiéval, Laboratoire d'Anthropologie Sociale, UMR 8210 Anthropologie et Histoire des Mondes Antiques

\section{Référence électronique}

Sophie Moiroux, «Les objets de Jimmie Durham en conversation : au défi de l'art contemporain », Images Re-vues [En ligne], 4 | 2007, document 5, mis en ligne le 01 janvier 2007, consulté le 31 janvier 2021. URL : http://journals.openedition.org/imagesrevues/153 ; DOI : https://doi.org/10.4000/ imagesrevues. 153

Ce document a été généré automatiquement le 31 janvier 2021.

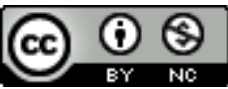

Images Re-vues est mise à disposition selon les termes de la Licence Creative Commons Attribution Pas d'Utilisation Commerciale 4.0 International. 


\title{
Les objets de Jimmie Durham en conversation : au défi de l'art contemporain
}

\author{
Sophie Moiroux
}

Les œuvres de Jimmie Durham, artiste contemporain d'origine cherokee, confondent systématiquement ceux qui les regardent. Elles ont certaines qualités étranges qui transforment des choses communes, bancales, cassées ou rejetées, en êtres mystérieux: des déclencheurs d'investigations. Dans la galerie d'art contemporain, ce sont des objets d'art.

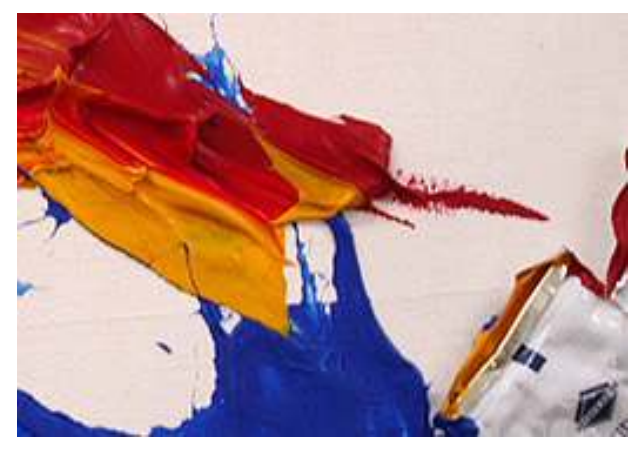
Nous allons voir qu'ils sont construits selon plusieurs cadres de lectures simultanés. Jimmie Durham les met en scène, ils deviennent en même temps des 'personnes' qui, vivantes, peuvent dialoguer avec les visiteurs. Une réflexion est engagée entre l'artiste, les œuvres et les spectateurs, qui voient leurs définitions et leur perspective sur le monde changer. L'artiste transforme les identités d'objets éclectiques, qu'il met en place et qu'il modifie dans ses œuvres. Il exemplifie ainsi une certaine approche des choses. Différents points de vue sont alors impliqués que nous découvrirons tour à tour en situant l'oeuvre. En regardant ici une exposition récente de Jimmie Durham nous envisagerons différentes conceptions des objets mises en jeu et comment, par imputations de subjectivités, des choses peuvent acquérir des qualités de personnes et converser avec les spectateurs. 


\section{A. sur la scène de la galerie}

\section{Différents aspects de l'œuvre}

L'œuvre peut être située tout d'abord sur les lieux-mêmes de son exposition : dans une perspective de l'art contemporain occidental. Investiguons les différents cadres de lecture que nous rencontrons dans la galerie. L'exposition considérée ici a été réalisée chez Barbara Wien, la galerie berlinoise de Jimmie Durham ${ }^{1}$. Elle a eu lieu du 28 avril au 1er juillet 2006, quelques mois avant le départ définitif de l'artiste de cette ville où il a vécu pendant huit ans. Pour cette occasion il a réalisé des œuvres avec les «Elements and Materials from my atelier in the Grunewald forest » (titre de l'exposition), c'est-àdire des objets trouvés (ou re-trouvés) dans son atelier, où ils se sont amassés pendant quatre ans. Cette mise en contexte est fournie au public dans le communiqué de presse disponible à l'entrée de la galerie sous forme de photocopies en anglais et en allemand ${ }^{2}$. Un article tiré d' ArtForum est également mis en évidence sur la table, aux côtés d'un portfolio de l'artiste, de copies de ses ouvrages, et de la liste des prix des œuvres exposées.

Dans l'histoire de l'art occidental, l'oeuvre de Jimmie Durham (né en 1940 en Arkansas, États-Unis) peut être rapprochée des travaux de Marcel Duchamp sur les « readymade », des surréalistes, de Fluxus (et notamment de Robert Filliou) ou de Arte Povera, entre autres. Jimmie Durham considère qu'en peignant il continue la conversation intellectuelle avec les peintres des siècles précédents ${ }^{3}$. Il se situe dans le monde de l'art occidental, dont il connaît parfaitement la culture. Son œuvre s'inscrit bien effectivement dans l'« histoire de l'art », et ses pièces sont présentes dans des collections et grands musées européens, des évènements artistiques internationaux (Biennale de Venise, biennale du Whitney, Documenta, etc.). Issu d'une famille de sculpteurs, Jimmie Durham a toujours fabriqué des objets; il a d'abord commencé par essayer de faire des investigations et des choses avec des matériaux. C'est bien plus tard, après son passage un peu par hasard à l'École des Beaux Arts de Genève où il est diplômé en sculpture, qu'il acquiert une connaissance de ce qu'est l'« art ». De même, c'est par les aléas des rencontres qu'il a commencé à vendre des pièces. Il conçoit alors qu'être un "artiste » est un engagement sérieux : il décide de faire de l'«art », mais pour sortir de ces limites, et interroger les appréhensions habituelles des objets. Il a en effet toujours " essayé de faire de son mieux pour fabriquer un discours excentrique de l'art »4. Il ne se considère "artiste» qu'en tant que cela est son (nécessaire) métier. Jimmie Durham est un « artiste contemporain ».

S'agissant de l'œuvre d'un artiste d'origine cherokee (identifiable en se référant à sa biographie, aux informations de la galerie, de la presse), nous pouvons être tentés de déceler des traces de sa culture dans son art, dans son aspect iconique par exemple. Jimmie Durham a toutefois toujours refusé de se trouver enfermé dans des stéréotypes (de l'« Indien », de l'« artiste indien », de l'« artiste », de l'« Histoire »), qu'il déconstruit continuellement dans son œuvre. Devant cette perception de son utilisation de peaux, os, perles, pierres, plumes, etc., il précise que «sauf si l'on parle d'une période très récente, ce ne sont assurément pas des matériaux amérindiens traditionnels au sens artistique du terme. En plus, ils ne nous appartiennent sûrement pas en propre $»^{5}$. En outre, " [p]rendre de nouvelles idées qui sont utiles est une activité très Cherokee $»^{6}$. Jimmie Durham refuse les catégorisations. Par ailleurs, la catégorie singularisante 
d'«art» n'existe traditionnellement pas pour les Cherokee: «La peinture (...) n'a besoin d'être intégrée dans la vie publique que dans les situations impliquant la praxis le donnant-donnant mutuel qui produit un réel changement. (...) Je crois que l'art devrait parler aux autres parties de la vie et ces autres parties devraient parler à l'art $»^{7}$. Il affirme qu'il s'agit plutôt pour lui d'« aider les gens à interpréter leur monde afin qu'ils puissent être capables de le changer de manière positive. Cela devrait être le but de toute activité humaine $»^{8}$.

Un important aspect de la biographie de Jimmie Durham est son engagement dans le monde, sa participation pour les droits des indigènes. Il a été activiste politique et dans les années 1970 il était membre actif de l' American Indian Movement qui s'est battu pour obtenir une amélioration de la situation des Indiens américains. Il a dirigé et représenté l' International Indian Treaty Council aux Nations Unies, pour la préparation du projet de la Déclaration des Nations Unies sur les Droits des Peuples Indigènes ${ }^{9}$. Cet engagement politique profond est très présent dans son travail, et il est par ailleurs visible dans ses nombreux textes politiques. Toutes les biographies et articles de presse mentionnent cet aspect de son œuvre et font partie de sa toile de fond. Le spectateur (tout comme certains critiques) peut réaliser cette mise en évidence de l'usage des stéréotypes dans son travail. En effet, les Indiens américains se sont retrouvés enfermés dans des réserves et des stéréotypes, expropriés de leurs terres et de leurs cultures. La nation Cherokee a été déplacée et en a particulièrement beaucoup souffert. Toutefois une des caractéristiques importantes de cette culture est sa capacité à être dynamique : «Le changement constant - l'adaptabilité (...) est une tradition que nos artistes ont particulièrement célébrée et ont utilisée pour faire bouger et fortifier nos sociétés (...). Nous [les artistes] pensons qu'en participant à tout dialogue moderne pertinent nous maintenons cette tradition $»^{10}$. L'artiste Jimmie Durham a un côté « occidental» qui fait partie de son identité propre de Cherokee ${ }^{11}$. Depuis 1994 il vit en ce qu'il nomme « Eurasie ». Il souligne qu'il a besoin d'être engagé intellectuellement là où il se trouve et en ce moment particulier. Ses expositions sont ainsi très souvent spécifiques à leur site. A la galerie Barbara Wien, Jimmie Durham parle de son atelier berlinois, de la forêt de Grünewald qui l'entoure, et de l'histoire de cet atelier : il a été construit par Hitler pour son sculpteur favori Arno Breker ${ }^{12}$ !

\section{Euvre située au-delà de l'« art »}


Fig.1.

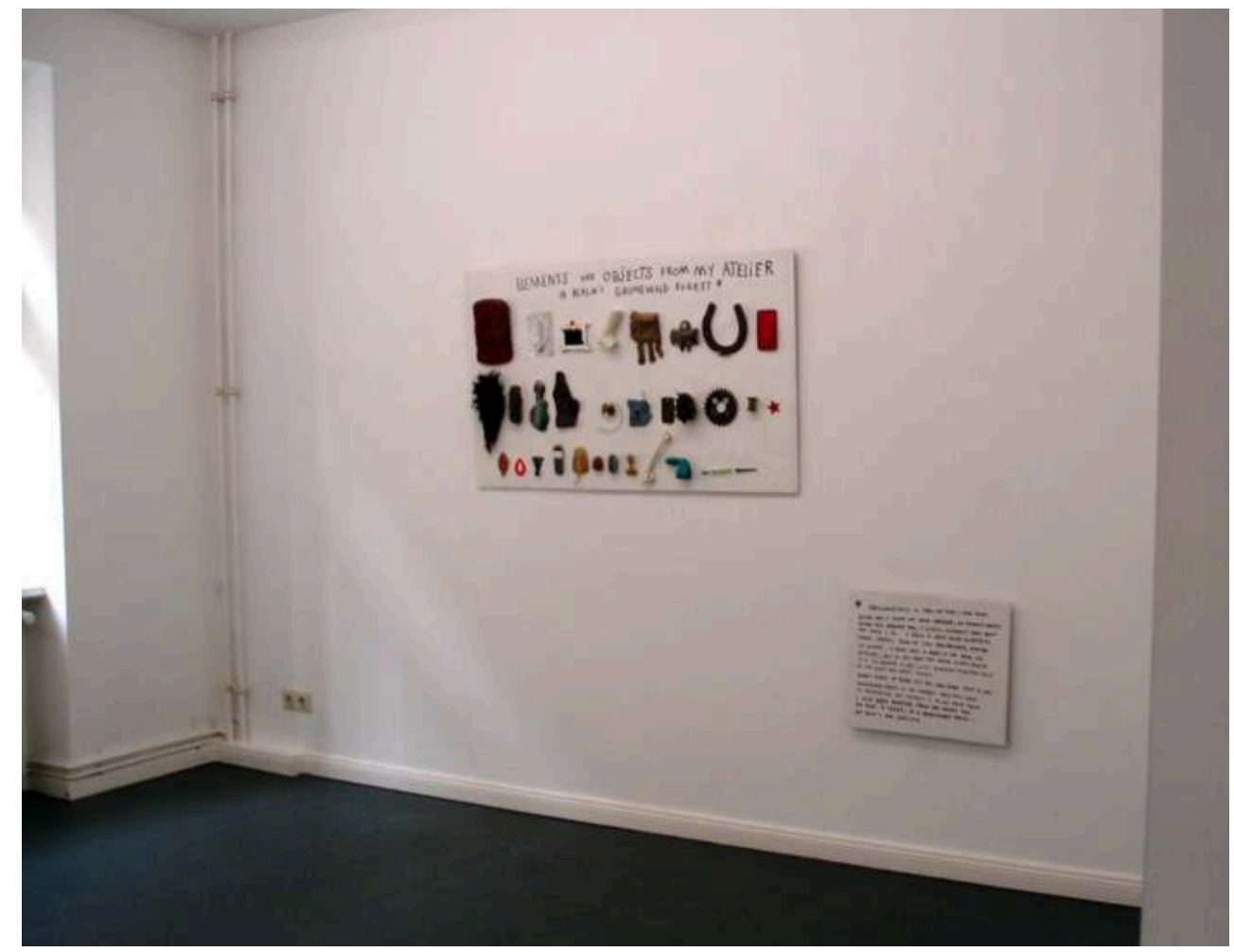

Elements and objects from my atelier in the Grunewald forest (2006), Galerie Barbara Wien, Berlin Photographie de l'auteure 
Fig.2.

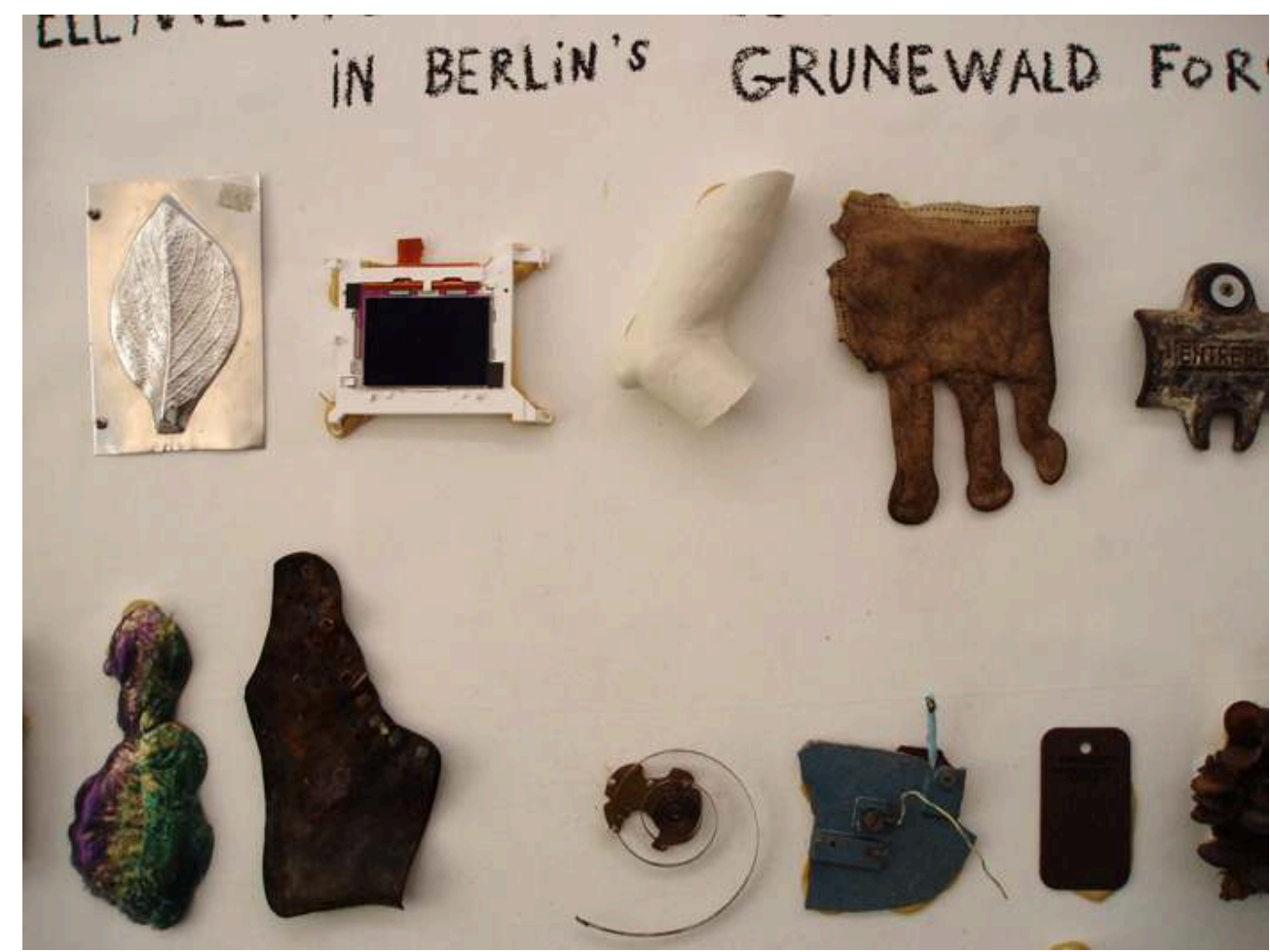

Elements and objects from my atelier in the Grunewald forest (2006), detail Photographie de l'auteure 


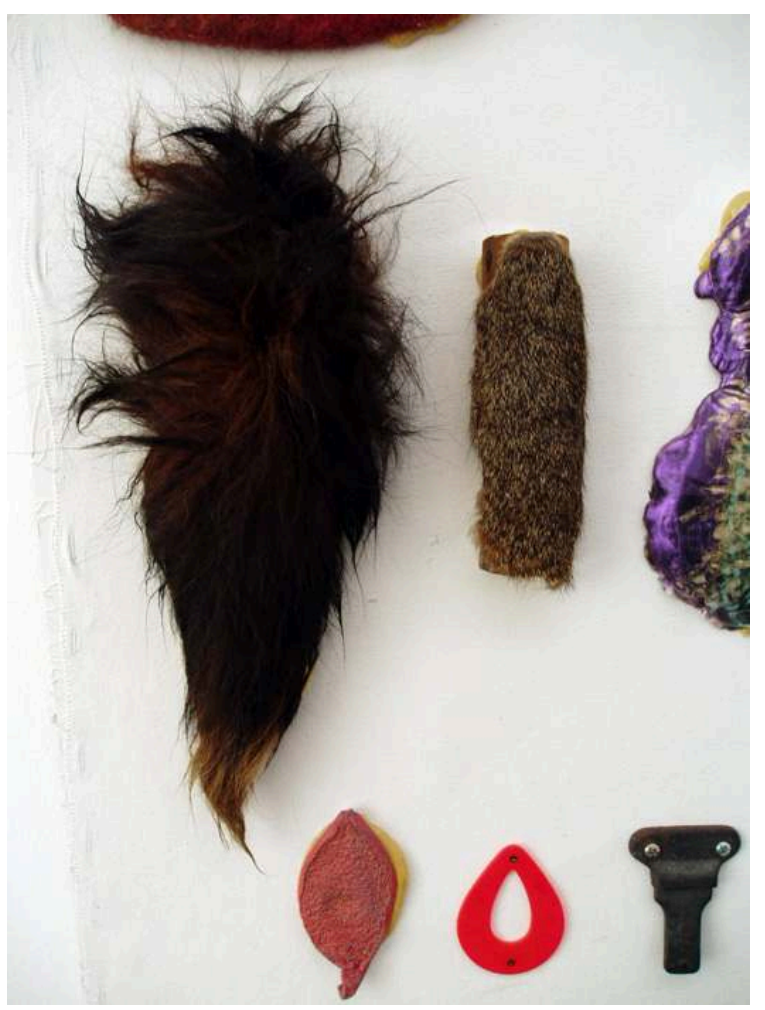

Elements and objects from my atelier in the Grunewald forest (2006), détail

Photographie de l'auteure

L'œuvre de l'artiste est ainsi mise en contexte dans les réseaux caractérisant l'art contemporain. Dans cette perspective, avec un oeil du monde de l'art occidental sur les choses, regardons de plus près les pièces exposées dans la galerie berlinoise - des aspects confondants apparaissent. On note tout d'abord que la distinction des différentes formes d'art est brisée (les pièces sont-elles des peintures ou des sculptures?) et que les matériaux utilisés ne sont généralement pas des matériaux «artistiques » ou bien, ils sont détournés de leur usage. Observons l'oeuvre-emblème de l'exposition : c'est une œuvre très spécifique au moment et à la situation et qui est placée dans la dernière salle de la galerie. Considérée comme un témoignage du travail de l'artiste à Berlin, Elements and objects from my atelier in the Grunewald forest (2006) (fig.1), comme l'annonce son titre, peut être perçue comme une collection d'objets alignés en présentation, provenant de son atelier ou comme une sorte de trophée, de blason ou encore de revue inventaire. Elle est composée de matériaux éclectiques (entre autres des fourrures, une seringue verte, une étoile en plastique rouge, un morceau de soulier), d'objets mis en exposition, de spécimens indéfinissables (figs. 2 et 3). D'autre part, nous pouvons lire Elements and objects from my atelier in the Grunewald forest comme un texte dont les mots et phrases, bien alignés horizontalement, sur trois lignes, peuvent être perçus ici comme des groupes d'objets. Toutefois, ces objets ne sont pas systématiquement reconnaissables et leur identité et leur fonction premières n'apparaissent pas toujours. Pourtant ces objets quotidiens sont trouvés sur place ou ont une résonance familière pour les visiteurs de la galerie. Leur définition est plus libre et plus ouverte à la réflexion. L'attention aux matériaux et aux éléments eux-mêmes est forcée. De plus, un astérisque attaché au titre nous 
apporte des précisions. La « note de bas de page » qui se trouve ici au pied du mur blanc de la salle d'exposition sur un petit cadre séparé, donne un sens à la lecture. Elle fournit des éléments biographiques de l'oeuvre et de l'artiste, sorte d'aspect vasarien pour l'histoire de l'art (fig. 4). Mais Jimmie Durham offre ces points d'informations plutôt pour désorienter le spectateur. Cette note ajoute un deuxième cadre de lecture. Le texte - « hitler and breker had, i guess, different ideas about art than i do. i seem to have more material than ideas" - notifie pour nous une divergence dans la perception d'« idées » dans l'oeuvre, depuis le même atelier : contrairement à Hitler ou Breker (qui construisaient des monuments nazis grandioses), Jimmie Durham propose de regarder de près les «matériaux ».

Fig.4.

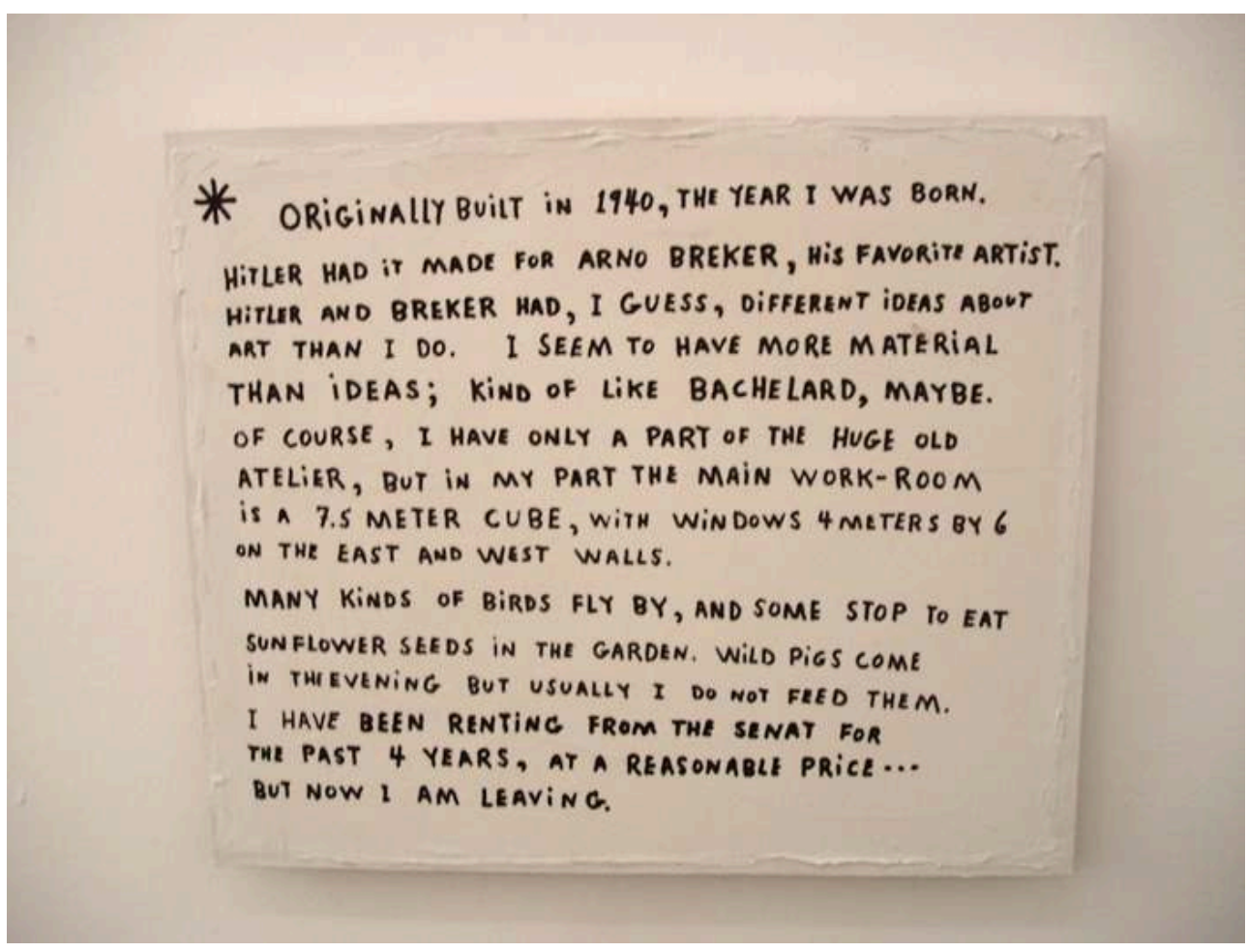

Elements and objects from my atelier in the Grunewald forest (2006), détail

Photographie de l'auteure

Cet exemple nous permet de distinguer deux manières d'appréhender une œuvre. Mais celles-ci semblent impossibles à réaliser indépendamment l'une de l'autre et sont paradoxales. Le matériau indéfini ne renvoie pas nécessairement à un référent connu. La lecture de l'œuvre est brouillée face aux matériaux individuels qu'elle ne comprend pas. Ces deux conceptions sont contradictoires l'une avec l'autre: fixité du signe, ou indécision de l'objet singulier. Au sein d'une même oeuvre, elles sont superposées et nous pouvons " lire " ses éléments (ou les lier en une communication) comme un texte qui se rapporte à lui-même. Les éléments et les matériaux ne sont pas des «signes ». Il s'agirait plutôt de concevoir un cadre de compréhension où se trouvent des "signes linguistiques » et un autre où il y a des " objets-personnes $»^{13}$. Ces derniers sont définis par leurs relations et doivent être appréhendés dans leur matérialité. Dans certains contextes et en voyant les choses au centre de relations entre les gens, nous pouvons comme Alfred Gell traiter les objets comme des « personnes » dotées d'intentionnalités. 
Les choses en effet sont définies par les réseaux de relations qui se déroulent autour d'elles comme c'est le cas avec une personne humaine, dont les intentionnalités peuvent d'ailleurs être inférées à partir de l'objet en tant $\mathrm{qu}^{\prime}$ «Indexe $»^{14}$. Les deux cadres sont intégrés : dans cette accumulation, les «signes » sont remplacés par les «objets». Nous voyons alors des objets d'art, qui font partie d'un tableau/une page. Nous voyons alors des objets d'art, qui font partie d'un tableau/une page. Mais ils rendent le spectateur confus. Sa perception est interpellée et accumule les différentes approches. La possibilité d'une divergence entre le langage et l'oeuvre d'art qui n'est pas une illustration est ici rendue apparente. La conception d'« art » est questionnée par cet ensemble d'objets qui font partie de l'attaque de Jimmie Durham contre les catégories qui gouvernent et enferment les gens. L'artiste a expliqué qu'« Au temps des cathédrales l'art avait un boulot clair : il devait illustrer. Et il pouvait illustrer par la peinture ou par la sculpture. L'art servait le Narratif, nous pourrions dire. C'est probablement pourquoi tant de gens veulent encore que l'art signifie quelque chose d'exprimable dans le langage, et supposent qu'il est « sans signification » s'il ne le fait pas. L'art servait le Narratif, mais les artistes voulaient servir quelque chose d'autre, quelque chose comme l'art $»^{15}$. Tout en brouillant les catégories et les rôles des objets, Jimmie Durham situe son oeuvre d'une part dans le monde de l'art, d'autre part contre ce qui le caractérise normalement ${ }^{16}$. Les cadres de lecture se croisent dans la perspective du spectateur d'une galerie d'art occidentale.

Fig.5.

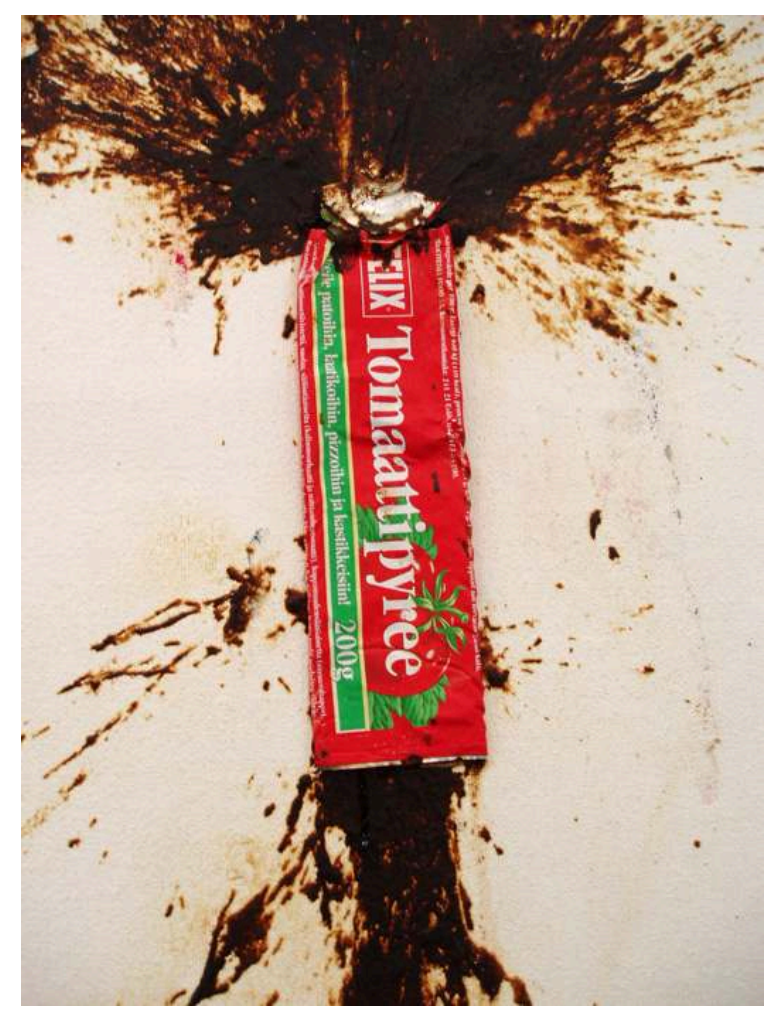

Where a stone fell on a tube of tomato sauce (1997), détail Photographie de l'auteure 
Fig.6.

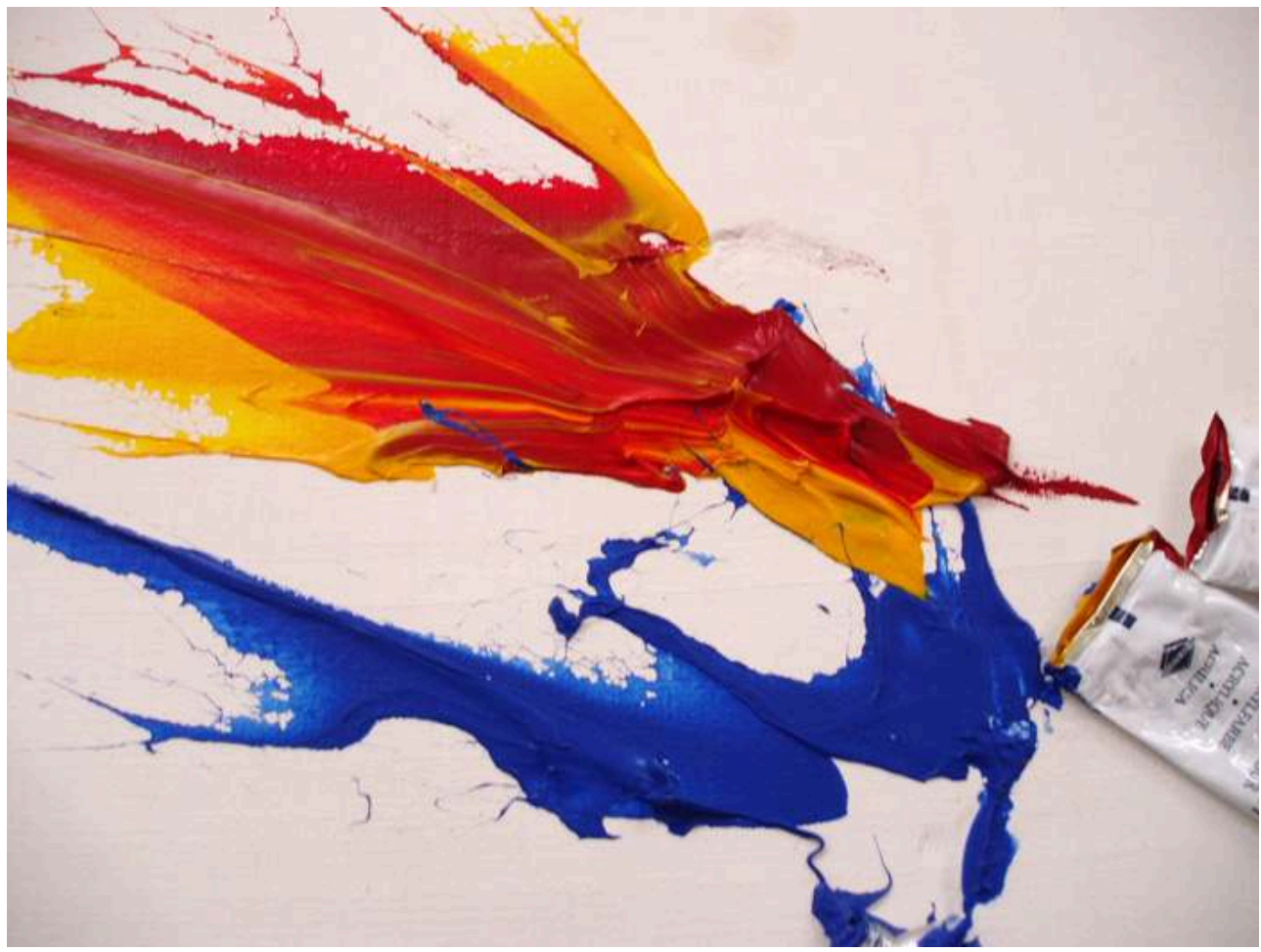

Where a heavy stone opened red, yellow and blue paint (1997), détail

Photographie de l'auteure

Fig.7.

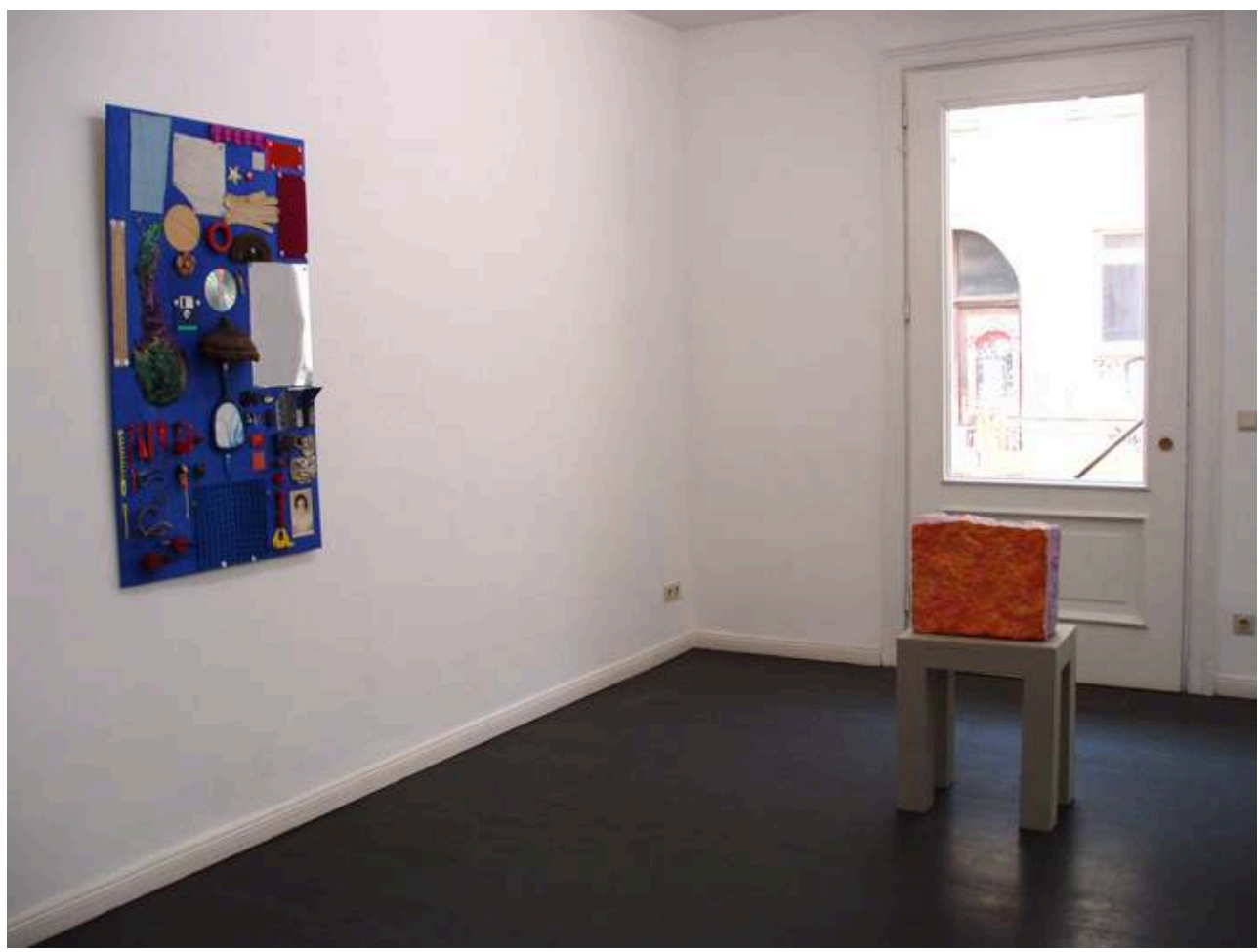

Stone rejected by the Builder I (2006) et 16 red things and a pecan (2006), Galerie Barbara Wien, Berlin Photographie de l'auteure 
Toutefois les identités des éléments impliqués dans l'oeuvre sont complexifiées. Les choses en effet sont comprises dans des réseaux de relations entre des gens et d'autres choses, qui constituent leur identité. Selon Gell, les objets-» Indexes » sont des traces d'événement ou d'objectifications d'intentionnalités. Nous pouvons alors reconstruire mentalement en une séquence d'actions, les « histoires » de leur origine et faire une "abduction » ou « inférence causale » sur les intentions ou capacités de ce qui les a produit. En suivant cette méthode indiciaire, nous pouvons en remontant les pistes partant des objets présents, percevoir et tenter d'identifier les entités comprises dans les réseaux relationnels qui les entourent et qui peuvent être différents suivant le point de vue. Jimmie Durham lui-même incite à l'investigation. Dans ses œuvres, nous voyons par exemple que l'«Artiste» (un des termes analytiques de Gell pour ordonner les entités en relations avec l'Indexe) n'est pas seulement Jimmie Durham. C'est une pierre qui a produit les tableaux (plus anciens) présentés à l'entrée de la galerie, Where a stone fell on a tube of tomato sauce (1997) (fig. 5) et Where a heavy stone opened red, yellow and blue paint (1997) composées de tubes écrasés par une masse dont l'impact est visible sur la toile (fig. 6). Dans le cas de Stone rejected by the Builder I et II (2006) (figs. 7 et 9), blocs de pierre peints, déposés comme des sculptures sur des socles, l'Artiste est également Arno Breker, invoqué dans le fait que, bien qu'il ne les ait pas sculptés en statues, il en ait probablement eu l'intention. En effet, ayant lu la note au bas du mur d' Elements and Materials from my atelier in the Grunewald forest, ou encore le Communiqué de Presse, nous réalisons que ces blocs proviennent de l'atelier de Breker et comprenons qu'ils ont été " rejetés par le constructeur ", le sculpteur nazi lui-même. Des sens distincts sont ainsi superposés. La transformation la plus récente imposée aux pièces par l'Artiste Jimmie Durham confère à ces dernières un sens nouveau, dissimulant l'autre, le rendant étrange lorsqu'il parvient à réapparaitre. Il a en effet peint l'une d'elles avec des couleurs enfantines et vives, pour qu'elle ait l'air " silly ». Les pierres ont changé d'identité. Le concept traditionnel d'artiste individuel et créateur est ainsi remis en questions. Tout en se situant dans la tradition duchampienne, en reconnaissant une conception de la relation matière/forme «selon Jung et Platon $\aleph^{17}$, Jimmie Durham conçoit l'histoire en tant que processus, « une succession de transitions et de contradictions - plutôt que comme les 'grands évènements' isolés et les 'découvertes' dont parlent les livres d'histoire ${ }^{18}$. Accumulés dans un même objet, ces aspects, retrouvés dans les éléments de Jimmie Durham, reflètent son engagement contre les catégorisations des colonisateurs. De plus, il a souligné il y a longtemps qu'il était essentiellement "constamment en train de rechercher une importance, une réalité aux choses ${ }^{19}{ }^{19}$ c'est-à-dire une attention pour les choses en elles-mêmes. Ceci implique une grande complexification des situations et multiples contextes qui sont définis par rapport aux relations sociales engagées autour des choses. Une compréhension des rôles des objets d'« art ", de leur capacité à agir dans un processus et un cadre plus largement social pourra alors être saisie en les analysant. Nous verrons alors les objets comme des "personnes" définies par les réseaux de relations comprenant ces éléments. De plus, une autre approche apparait qui considère les matériaux et éléments en tant que tels. Ajoutons que les identités des œuvres sont rendues plus accessibles au moyen non seulement des divers textes explicatifs proposés aux spectateurs, mais aussi par leur mise en relations avec les autres œuvres de l'exposition communiquant entre elles sur des murs adjacents dans la galerie. Dans ce contexte de l'art occidental, l'artiste a introduit un autre niveau de perception : il attire l'attention vers un point de vue basé sur les matériaux et sur les 
relations (matériaux entre eux, et avec l'artiste et les spectateurs), plutôt que sur les « idées ». Nous allons maintenant considérer les objets comme des « personnes », en un certain sens étonnamment « vivants ».

\section{B. mise en scène par Jimmie Durham}

\section{Jeux de l'artiste et vies des objets}

Sculpteur-né, Jimmie Durham dit qu'il a «fabriqué des objets avant d'apprendre à parler $»^{20}$. Il en a fait des jouets qui « impliquaient une vie fantaisiste très complexe » et il nous dit que "n'importe quel objet que je pouvais manipuler était sous la menace constante d'être transformé pour jouer un rôle dans mon monde (...). Je fabriquais ma propre société dans laquelle j'avais un rôle important. Quelque chose dans ma constitution me poussait à construire cette société avec des objets matériels que je pouvais changer d'une façon ou d'une autre; j'avais juste un " penchant» pour me lier avec les objets et les formes. Je suppose que cela est le cas pour beaucoup de sculpteurs ${ }^{21}$. Il a ainsi développé une approche particulière des objets et des matériaux. Une relation personnelle tout d'abord: «constamment, je parle avec les objets, je joue avec les objets, avec les relations qu'il y a entre eux, moi, et le reste du monde ${ }^{22}$. Réalisant des œuvres d'art, Jimmie Durham est inspiré par les objets euxmêmes: "Lorsque je vois un morceau de bois, un crâne de chien, une bouteille en plastique, je sens qu'il y a une connexion. Chacun de ces objets a une histoire politique et matérielle semblable à la mienne $»^{23}$. Il écoute les histoires des objets. De plus, il établit une sorte de "connexion directe avec les choses réelles et les processus du monde $»^{24}$. Il apparaît ici encore cette attraction, cette volonté d'utiliser ce qui se trouve sous sa main, qui permet de situer les choses - ceci étant un aspect important de leur identité. Face à elles, l'artiste est actif, il esquisse des réponses, continue un dialogue actuel. «Les objets se présentent d'eux-mêmes; j'ai donc (dans une certaine mesure) la responsabilité de me présenter aussi en retour $»^{25}$. 
Fig.8.

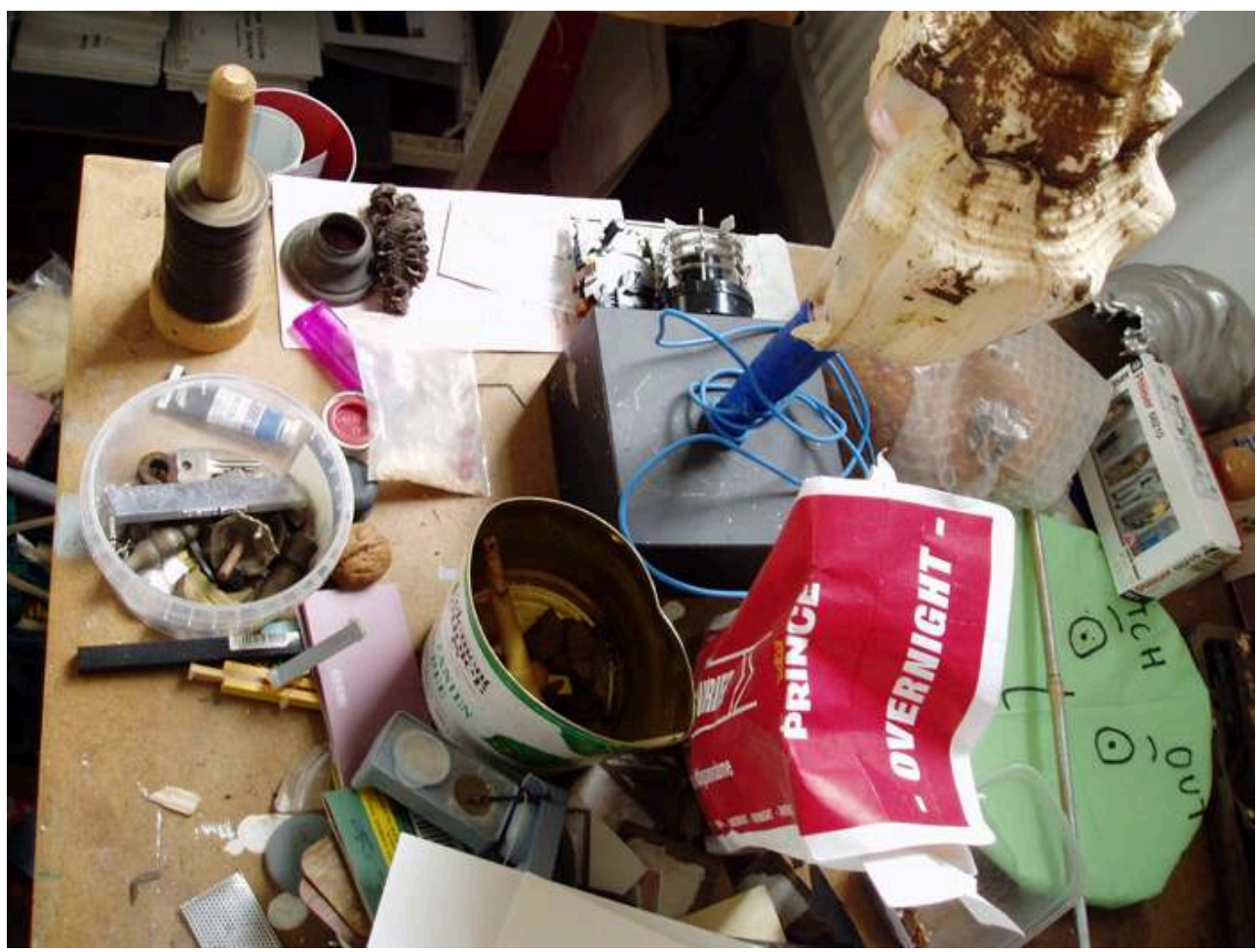

Photographie de l'atelier de Jimmie Durham, Atelier Breker, Berlin (avril 2006)

Photographie de l'auteure

Quant à eux, les objets proposent des questions, sans donner de réponses définitives : ils engagent le dialogue ${ }^{26}$. En effet, indéfinissables, incatégorisables, ils poursuivent une conversation quant à leur identité, leur histoire, leur « vie ». Jimmie Durham " invite [les objets qu'il a trouvés, par exemple lors de longues promenades] dans sa famille, et dans son studio et puis, il joue avec eux et puis, les renvoie dans le monde, d'une nouvelle manière $»^{27}$ (fig. 8). D'une part, les objets apparaissent avoir une " histoire " dans le sens de «biographie» ou de "point de vue». Différentes références temporelles sont accumulées dans les éléments et dans l'oeuvre, qui en tant que traces de ces épisodes rappellent leur définition (passée). Les matériaux maintiennent leur présence propre. L'artiste ne les force pas à devenir quelque chose de totalement nouveau: une oeuvre d'art originale, issue de son pouvoir de créateur. Il laisse apparentes leurs propriétés physiques: matériaux, formes sont respectés, et leur "vie» passée n'est pas effacée. Même la colle et les clous utilisés pour fixer ne trompent pas le spectateur sur la constitution de l'oeuvre d'art, cette stratégie permet aussi de concevoir le travail de l'Artiste Jimmie Durham. C'est grâce à ces traces laissées que nous pouvons reconstituer une vie des objets, leurs histoires, et leur attribuer des intentionnalités . Ceci est particulièrement frappant dans le cas des "objets trouvés ", et dans le fait que ses pièces n'ont jamais l'apparence d'être achevées. Elles restent liées à d'autres éléments et d'autres contextes dans le temps et dans l'espace. Nous avons vu ceci dans le cas de Stone rejected by the Builder I et II . Les objets sont ainsi plutôt transformés : ils acquièrent donc une nouvelle vie en continuation avec l'« ancienne " encore présente et complétée par une transformation effectuée par l'Artiste. Jimmie Durham exerce une "négociation", un dialogue à double sens avec ses matériaux. Par exemple, il présente un «portrait» de champignon A Mushroom from the 
Grunewald (2006) (fig. 9). Le champignon est placé au centre du tableau à fond blanc nacré, accompagné seulement d'un astérisque-étoile. Dans la " note ${ }^{28}$, l'artiste nous raconte l'histoire de sa découverte et nous propose de l'identifier par son nom scientifique : il pourrait s'agir de " 'destroying angel' (amanita virosa) but it might be instead the 'deceiver' laccaria laccata) ». Pour cela il a suivi des ouvrages de références en la matière, mais de façon comique. Puis il nous explique le processus de fabrication de cette pièce d'art : la conservation du champignon, la composition de la colle utilisée. Il nous donne ainsi des indications biographiques sur l'oeuvre, tout en soulignant sa matérialité et ses qualités physiques. Dans cette «note ", commentant son apparence, l'artiste attire aussi l'attention sur le fait que le champignon et le spectateur, bien que différents dans leur aspect visuel, devraient pouvoir s'entendre dans une même conversation - entre entités vivantes et de même intelligence : « yes, it does look a bit strange ; nevertheless. you are also a bit strange ». Cette mise en relation permet de situer l'objet d'art dans un réseau constitué de « personnes » dotées d'intentionnalités.

Fig.9.

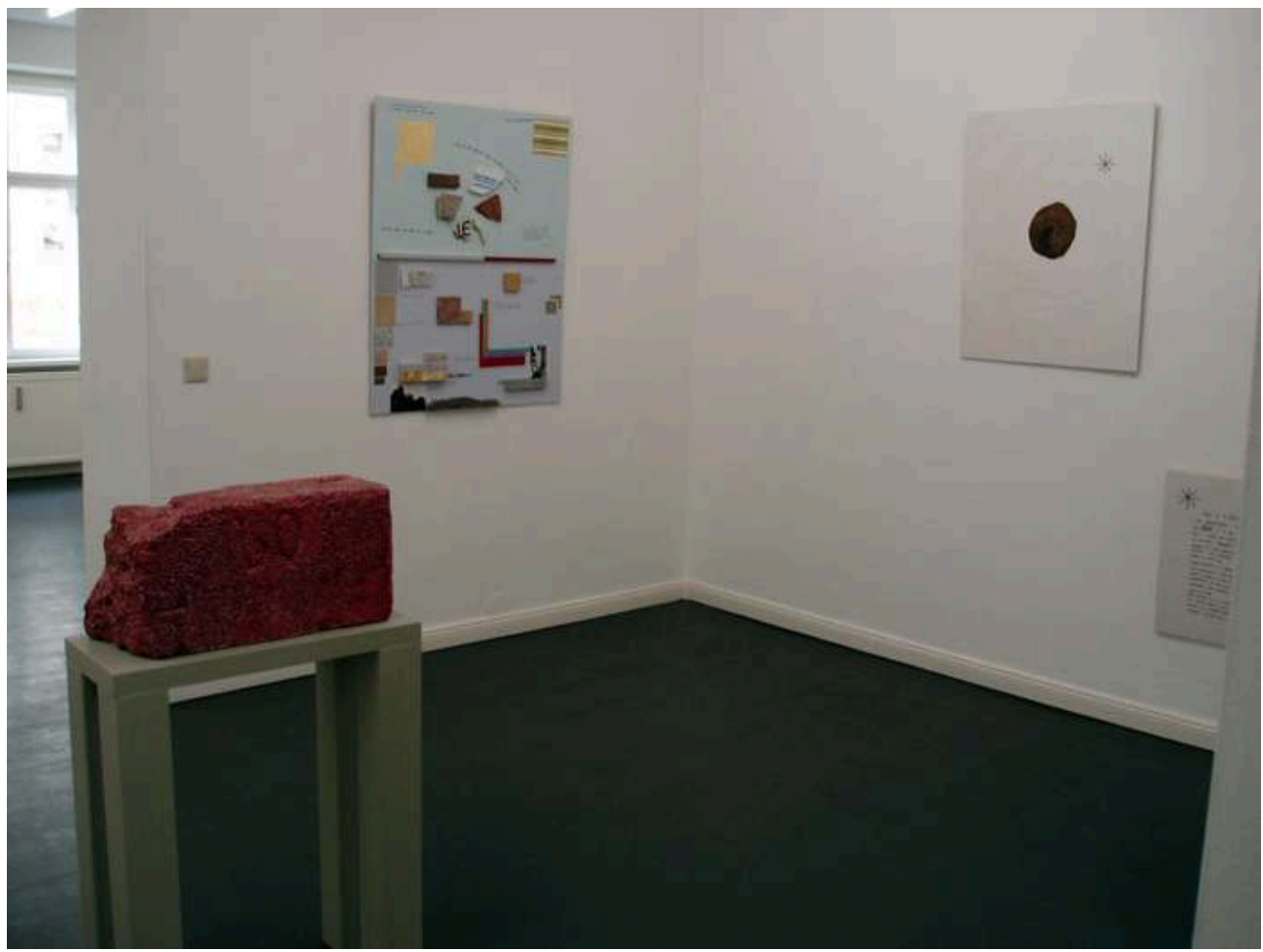

A Mushroom from the Grunewald (2006), They Almost Fit (2006), et Stone rejected by the Builder II (2006), Galerie Barbara Wien, Berlin

Photographie de l'auteure

Pour approcher ces objets, il est alors intéressant de considérer une autre manière de les concevoir. Dans les cosmologies amérindiennes, les différents êtres ontologiques ou espèces de sujets perçoivent les choses de la même façon: "le point de référence commun pour tous les êtres de la nature » est «l'humanité en tant que condition ${ }^{29}$. Chaque entité disant 'Je', se trouve ainsi dans un système de relations identique à celui de la perspective humaine. Eduardo Viveiros de Castro propose de concevoir que l'«âme» ou «jeu de capacités cognitives et volitionnaires» est comprise comme commune à tous les sujets, et que la différence ontologique (et référentielle) entre les 
êtres se trouve alors « inscrite dans les corps, [le corps étant] un système dispositionnel d'affectabilité $»^{30}$. Nous pouvons ainsi considérer l'«affectabilité » du «corps » d'une chose comme une capacité d'entretenir des relations avec son entourage - Viveiros de Castro la définit comme « ses capacités à affecter et à être affectée par d'autres corps ». Nous proposons de comprendre ici de cette manière les qualités matérielles des objets. Ces relations constituent un certain monde, celui d'un objet par exemple; les étudier permet de le comprendre, de voir comment il s'y définit lui-même. Ce genre de relations peut alors servir à fournir une définition des objets en direction de ceux qui les regardent . Les êtres étant des points de vue (qui disent 'Je'), la question devient : " de quel monde (...) sont-ils le point de vue " ? $^{31}$. En face d'un objet, l'on doit s'attendre à faire face à une autre perception par un autre point de vue. Différents corps ayant différentes expériences du monde, différentes entités perçoivent alors différentes choses. De plus, pour une même chose, le critère définissant son concept est différent suivant le " point de vue $»^{32}$ ou " perspective». Nous pouvons ainsi percevoir les objets en tant qu' ils ont un point de vue sur le monde, qui leur est propre, et qui ainsi définit leur identité. Ce monde, nous-mêmes le comprenons autrement, selon d'autres critères. Ces différents mondes et les identités des choses dans ces mondes sont construits selon la mise en place de réseaux de relations qui situent et définissent les choses. Dans le cas d'un objet $d$ ' art , considéré dans différents mondes ou selon différentes perspectives ou "points de vue ", il s'agit de voir comment l'artiste met en relief une certaine définition de cet objet - en même temps qu'il acquiert une intentionnalité aux côtés d'autres choses avec lesquelles il est mis en relation, et peut alors se définir en 'Je' intentionnel.

\section{Relations entre les choses}

Voyons comment sont conçues les définitions des objets. Jimmie Durham considère que la fonction des sculpteurs n'est pas de « créer » mais de "changer les objets ». Notons que la transformation d'identités des choses ne s'exerce pas nécessairement au niveau physique, mais comprend le contexte, ou la possibilité d'autres points de vue. Elle peut aussi être soulignée par l'aspect pragmatique qui produit de nouvelles relations autour d'elles et donc change leur définition ${ }^{33}$. Cette idée de transformation concertée est intéressante : elle produit la possibilité d'un dialogue dynamique entre l'artiste et les objets, mais aussi entre les objets eux-mêmes (et puis avec les spectateurs). Nous avons vu que Jimmie Durham était attiré par les objets. Mais ensuite : comment les objets se retrouvent-ils ensemble? Comment les relations sont-elles établies entre eux? Une autre manière que l'artiste a de les choisir suit le fait que, selon lui, les objets collectés en « appellent » d'autres ${ }^{34}$, en une sorte de complicité.

De la perspective d'un monde différent, il s'agit tout d'abord de voir quels types de connexions les objets sont susceptibles de constituer et qui leur sont établies par leur environnement, et comment ceci permet de comprendre leurs identités et leur imputation d'intentionnalité. Considérons une approche des objets différente de celle que nous avons vue précédemment dans le cadre de la galerie et qui implique une autre perspective. Ces types de relations peuvent apparaitre entre des entités non-humaines mais étant de cette manière définies comme des personnes dotées d'intentionnalités. Jimmie Durham a expliqué : «Lorsque nous faisons une œuvre sur du papier nous ne pouvons oublier le processus de fabrication du papier, le fait des arbres sur notre terre. (...) Quels langages sémiologiques pouvons-nous exposer [avec chaque œuvre d'art]? 
Les langages parlent au travers des objets. Une bouteille de whisky a un usage artistique tout aussi valide que n'importe quel autre objet. Bien sûr, nous écoutons les conversations de la bouteille lorsque nous considérons les buts artistiques. Ce serait une sorte de péché que de duper la bouteille pour la faire mentir: question d'intercepter le dialogue universel si nécessaire à notre survie. On peut utiliser une bouteille pour jouer des tours, ou pour raconter des blagues, ou pour faire des combinaisons inattendues, mais nous ne la forcerions pas dans de fausses positions ${ }^{35}$. Considérant l'hypothèse selon laquelle les propriétés physiques et relationnelles d'une chose sont la base de son identité, il est important d'observer attentivement ces qualités et ces relations pour comprendre l'intentionnalité qui la rend active. Le principe de mise en relation que nous venons d'apercevoir entre diverses choses et l'artiste, mais aussi en principe tout être humain, peut être appliqué aux objets transformés en éléments d'œuvres d'art, avec un respect pour leurs propriétés, changées ou non, et avec l'ajout de texte, dit ou écrit. Insérés dans un certain contexte alors construit et appelés à communiquer, à raconter leur histoire, à se présenter, ils deviennent vivants. De plus, il a souligné que ses œuvres sont fondées sur un art de connexions et d'attention à la "réalité des choses »: il est nécessaire pour lui de considérer une "'qualité' des choses, la qualité des actions, des changements, des systèmes, etc. ». « Nous n'acceptons pas les idées de 'développement' ou de 'croissance économique' " dit-il, «à moins que nous puissions voir clairement tant à long terme qu'à court terme les bénéfices qu'ils fourniront aux êtres humains [...]. Le concept nous permet de choisir. Nous acceptons les téléphones. Nous n'acceptons pas le DDT ou le napalm $»^{36}$ - des liens directs (et constitutifs de leur définition) entre objets, potentiellement intentionnels, et humains, fondent leur usage, et par extension leur insertion dans des œuvres d'art.

\section{Matériaux en scène}

Des relations sont mises en place avec les choses, impliquant matériaux et textes. Jimmie Durham déclare «Je voudrais rendre art chaque chose individuelle existante, il n'y aurait pas un moment où l'on aurait à décider quoi garder et quoi jeter. Et, il me semble, l'on peut faire cette sorte de chose non-dictatoriale en fabriquant des choses qui n'ont rien à voir avec de l'artisanat, qui rejoignent juste intellectuellement notre monde physique normal ${ }^{37}$. Les choses éclectiques - matériaux organiques (peaux, noix, os), matériaux manufacturés (tissus, laine, plastiques), pierres, morceaux d'objets identifiables ou non, objets entiers (poinçon, clou, miroir, disquette, gant), formes diverses, photographies, sont tous considérés comme également importants. Il n'y a pas de hiérarchie entre eux. Les mots eux-mêmes sont aussi conçus comme des "matériaux $»^{38}$. Jimmie Durham, qui écrit également des poèmes, traite les objets comme les mots, comme des matériaux : il joue avec eux quels qu'ils soient, et les met en relation. Il écrit : « je rassemble les choses et les mets dans mon studio. Alors je peux les utiliser dans des œuvres, simplement comme je pourrais utiliser des mots dans des phrases $»^{39}$. Il assemble ainsi différents cadres de lecture, utilisant les concepts de différents mondes. 
Fig. 10.

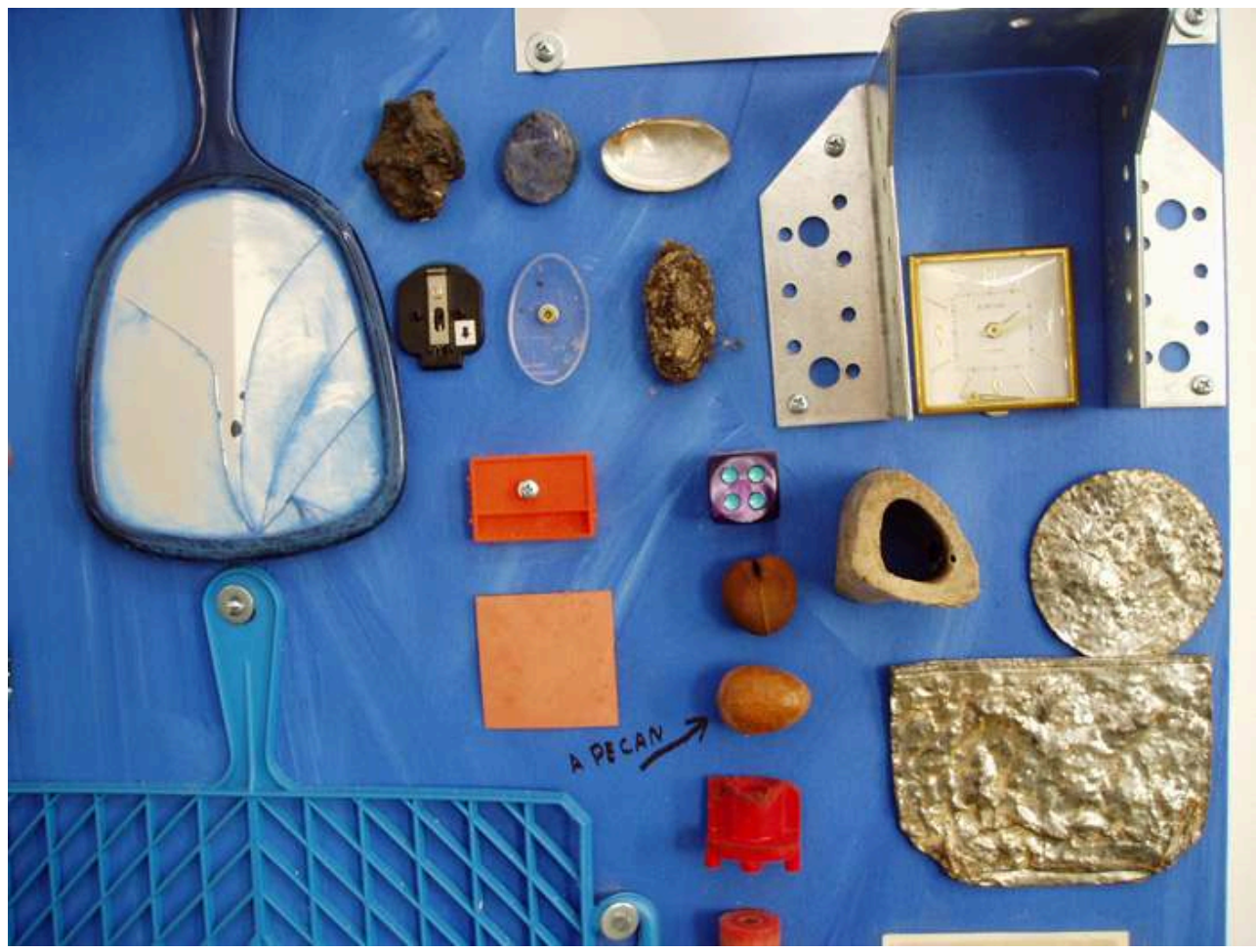

16 red things and a pecan (2006), détail

Photographie de l'auteure

Fig. 11.

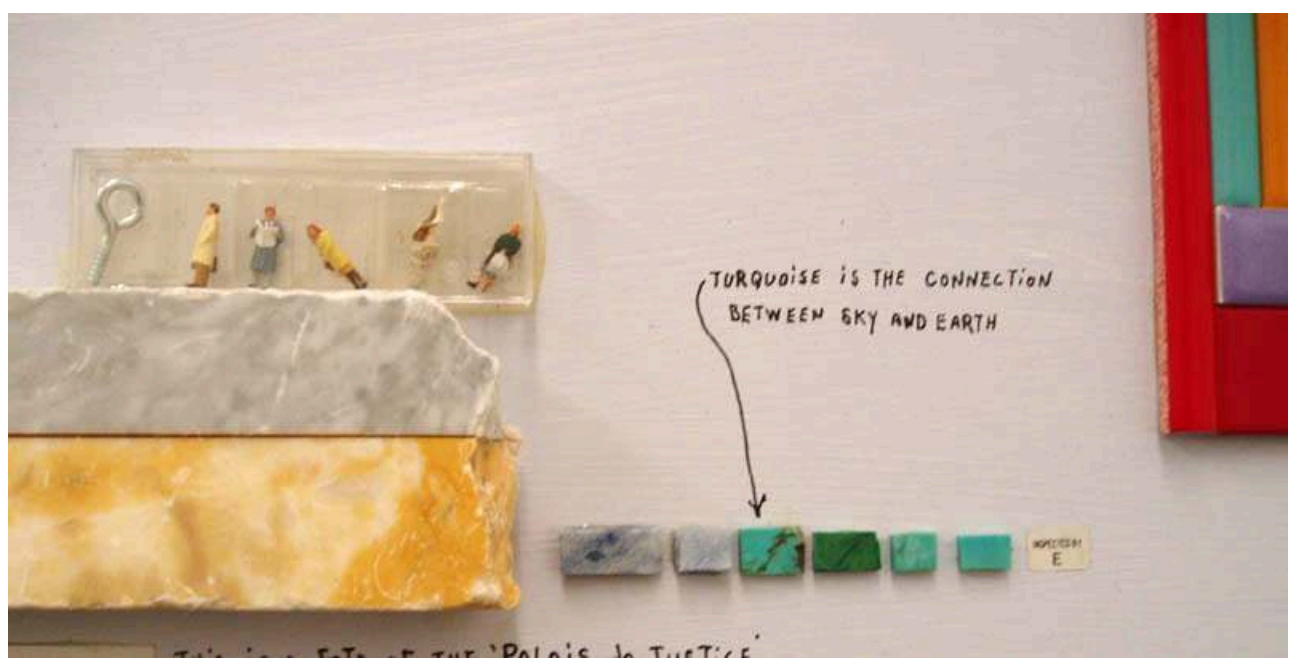

They Almost Fit (2006), détail

Photographie de l'auteure 
Fig. 12

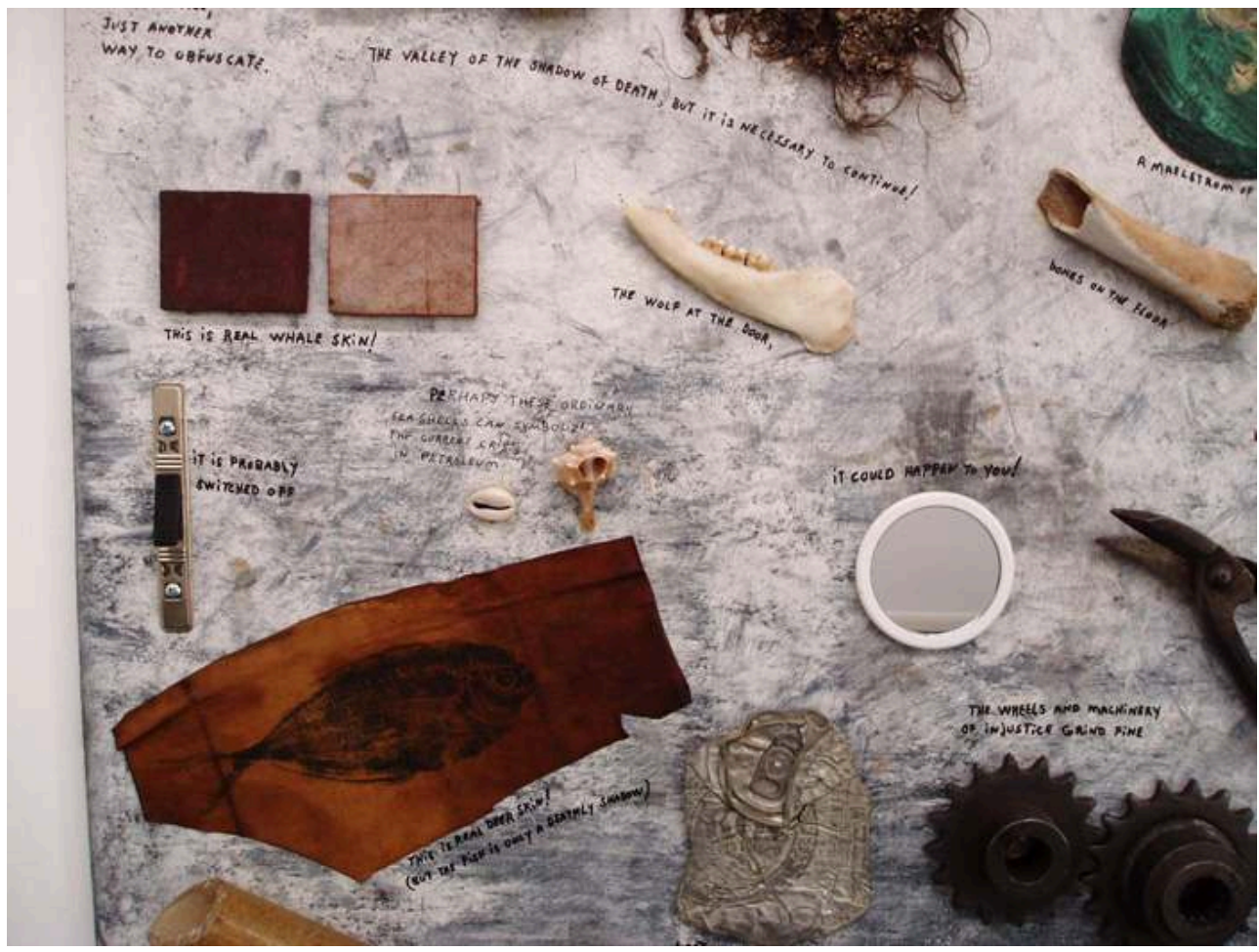

Snake eyes! (2006), détail

Photographie de l'auteure

Fig. 13

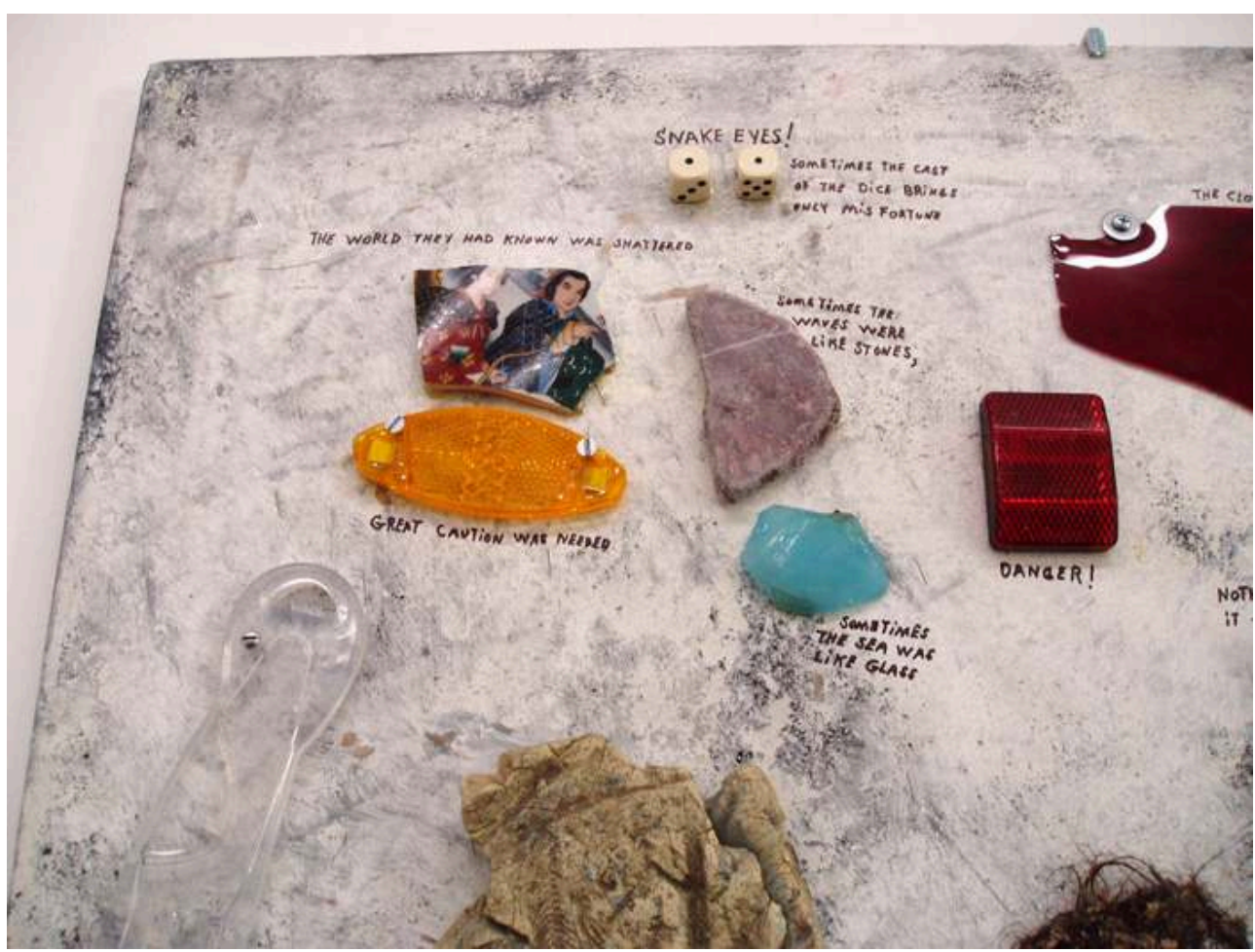

Snake eyes!(2006), détail

Photographie de l'auteure 
Fig.14.

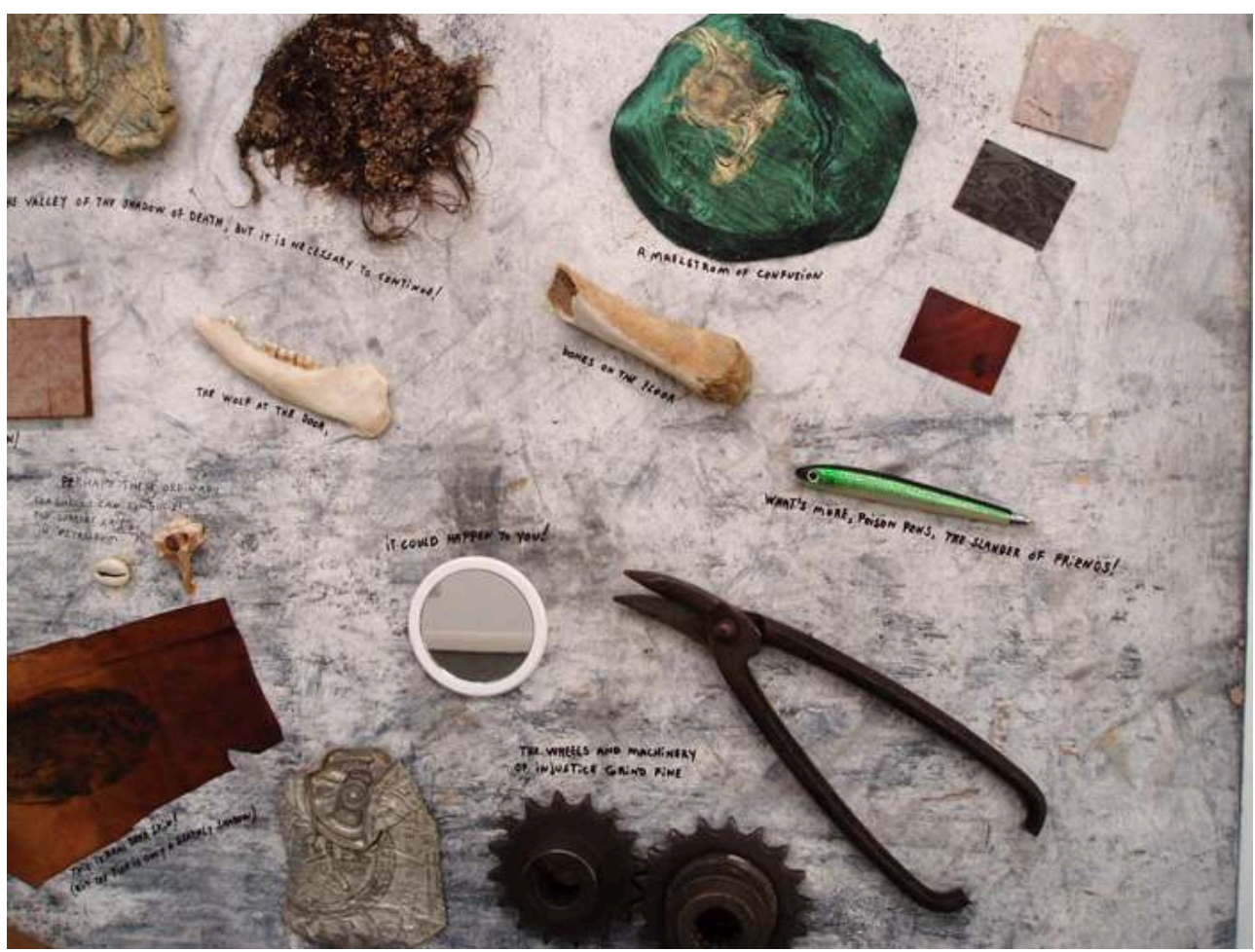

Snake eyes ! (2006), détail

Photographie de l'auteure 
Fig.15.

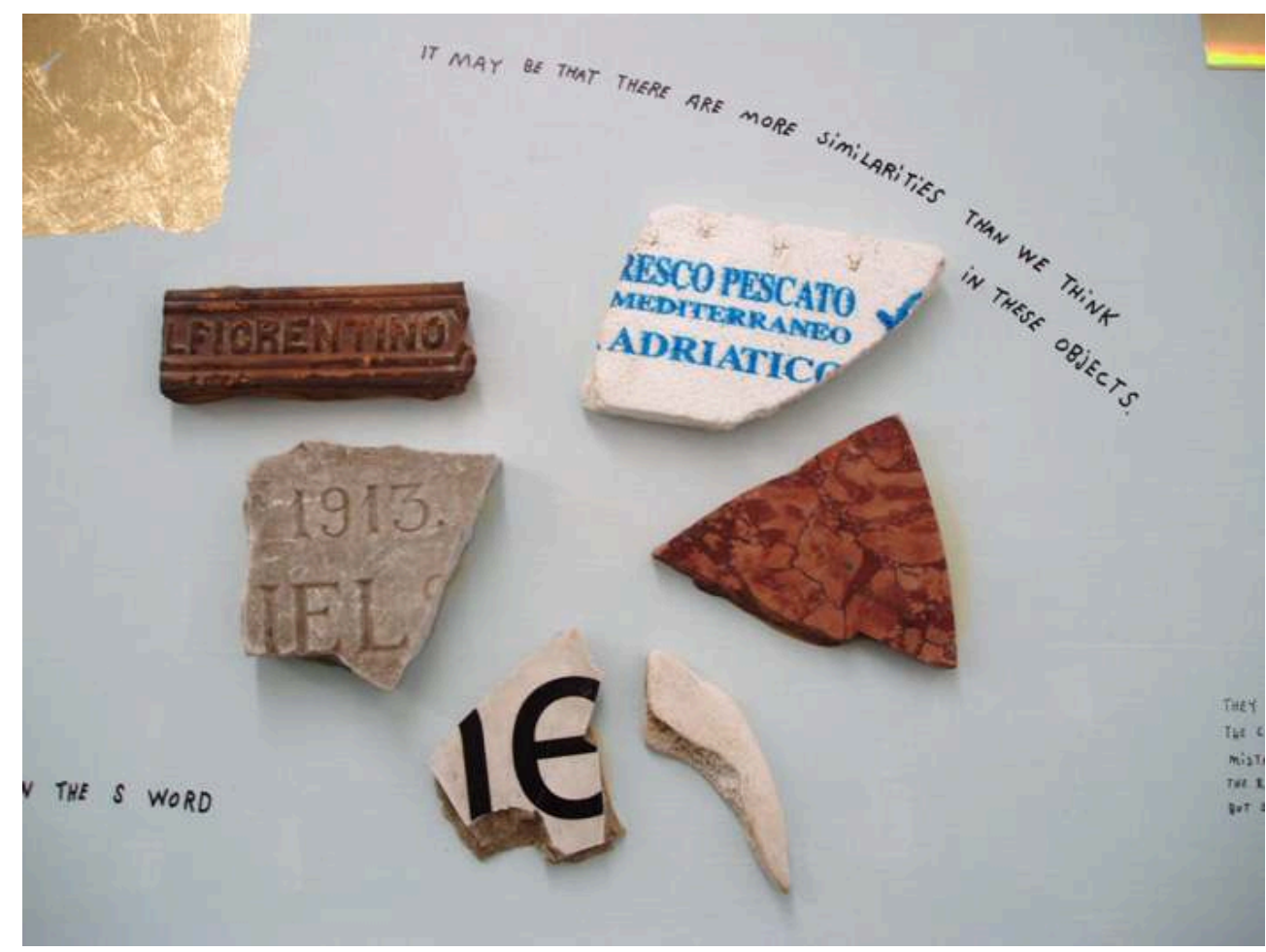

They Almost Fit (2006), détail

Photographie de l'auteure

L'artiste prise les connexions entre mots et objets. Il dit: «Je n'aime pas le langage (mais il ne s'en suit pas que je recommande son abandon ou son mauvais usage - bien au contraire), mais j'aime les mots. (Pardonnez s'il vous plait cette digression, pour une certaine raison nous parlons comme s'il y avait une connexion entre les mots et les objets. L'une des propriétés intéressantes tant des mots que des objets est qu'ils se repoussent les uns les autres. Souvent j'aime les combiner dans des œuvres d'art en les clouant ou collant ensemble à cause de la vibration que cela cause) $»^{40}$. Le texte, qui produit normalement le sens, est mis en relation avec les objets pour leur donner une dimension transformationnelle supplémentaire, dans ses œuvres d'art. D'une part l'ajout de mots qui semble habituellement être un élément nécessaire à l'appréciation des choses est attaqué par exemple par un petit texte recopié dans They Almost Fit (2006) : " they laughed, and with this laughter the cracks widened, she realize her mistake then and xx knew that only the right word would save the situation, but she could not grasp it. alice munro ". L'artiste rajoute parfois des morceaux d'ouvrages littéraires également comme des matériaux. De plus, il inscrit souvent des sortes de définitions sous les objets, reliés avec une flèche : « a pecan » (fig. 10), « turquoise is the connection between sky and earth» (fig. 11). Certains commentaires en questionnent la validité ou la nécessité, renvoyant par ailleurs à d'autres contextes : «this is real whale skin!», «this is real deer skin! (but the fish is only a deathly shadow)» (fig. 12). Les matériaux accompagnant les textes - ou l'inverse - ne sont pas des illustrations ou des représentations mais des choses réelles: "sometimes the waves were like stones ", "sometimes the sea was like glass" sont notés respectivement sous un morceau de pierre et sous un morceau de verre (fig. 13). Les objets et les mots ont chacun une existence propre et indépendante. Chacun peut faire partie de jeux de mots/matériaux 
comme " a maelstrom of confusion » (fig. 14). Les titres sont également des éléments importants des pièces. Stone rejected by the Builder nous renseigne sur une partie de l'histoire de la pierre. They Almost Fit souligne la dichotomie entre les formes du Formalisme et l'assemblage d'objets qui ne collent pas comme l'on voudrait, et qui se retrouvent ainsi en tant que "particuliers » et peuvent donc discuter entre eux (fig. 15). Les objets et éléments apparaissent non pas génériques mais comme des individus. Jimmie Durham les met en relations tout en respectant leurs natures différentes : nous pouvons lire des phrases, faire des connections de formes entre les choses par exemple. De plus, un sens nouveau est créé par la juxtaposition de ces différents aspects: présence de l'objet-Indexe, matière, forme, texte, mots, qui se modifient mutuellement par leur assemblage. Des liens sont également établis avec d'autres fonctions, significations, ou histoires. Chacun des objets est considéré comme une personne et peut dialoguer. Ils ont des intentionnalités et des vies antérieures, et il s'agit de questionner ces êtres mystérieux situés dans un nouveau contexte, un nouveau réseau de relations. De cette manière Jimmie Durham leur fournit une scène où se présenter : celle de l'oeuvre et celle de la galerie et du monde de l'art contemporain. L'artiste dit : «j'éprouve un urgent besoin de récupérer les 'rebuts' $»^{41}$. Il veut leur rendre une place dans le monde en quelque sorte, dans certaines relations sociales, et en tant que luimême. Différentes communications sont ainsi établies, dans les textes et les matériaux, entre les objets et impliquant l'artiste.

\section{C. dialogues avec le spectateur}

\section{Approcher des objet-personnes}


Fig.16.

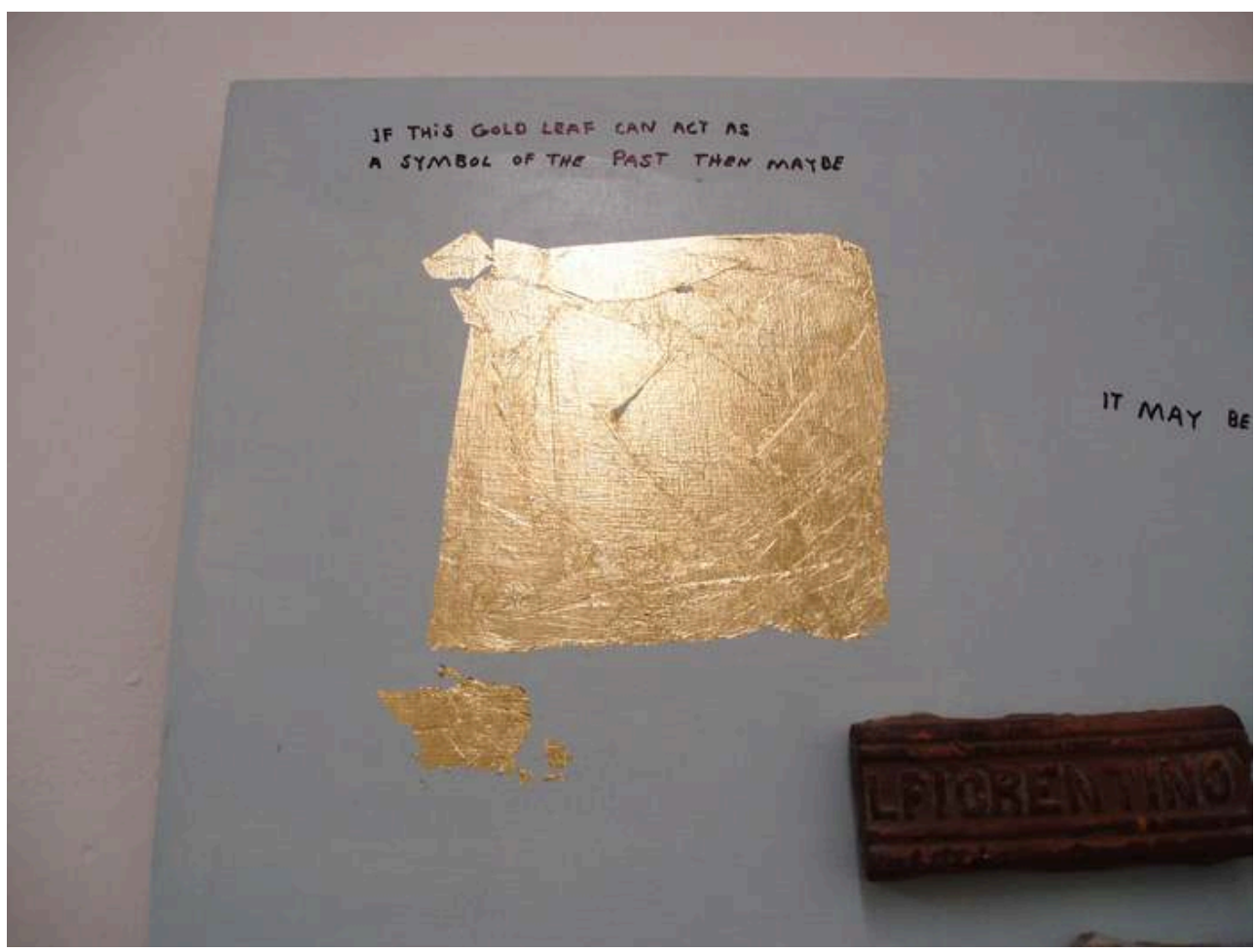

They Almost Fit (2006), détail

Photographie de l'auteure

Fig. 17.

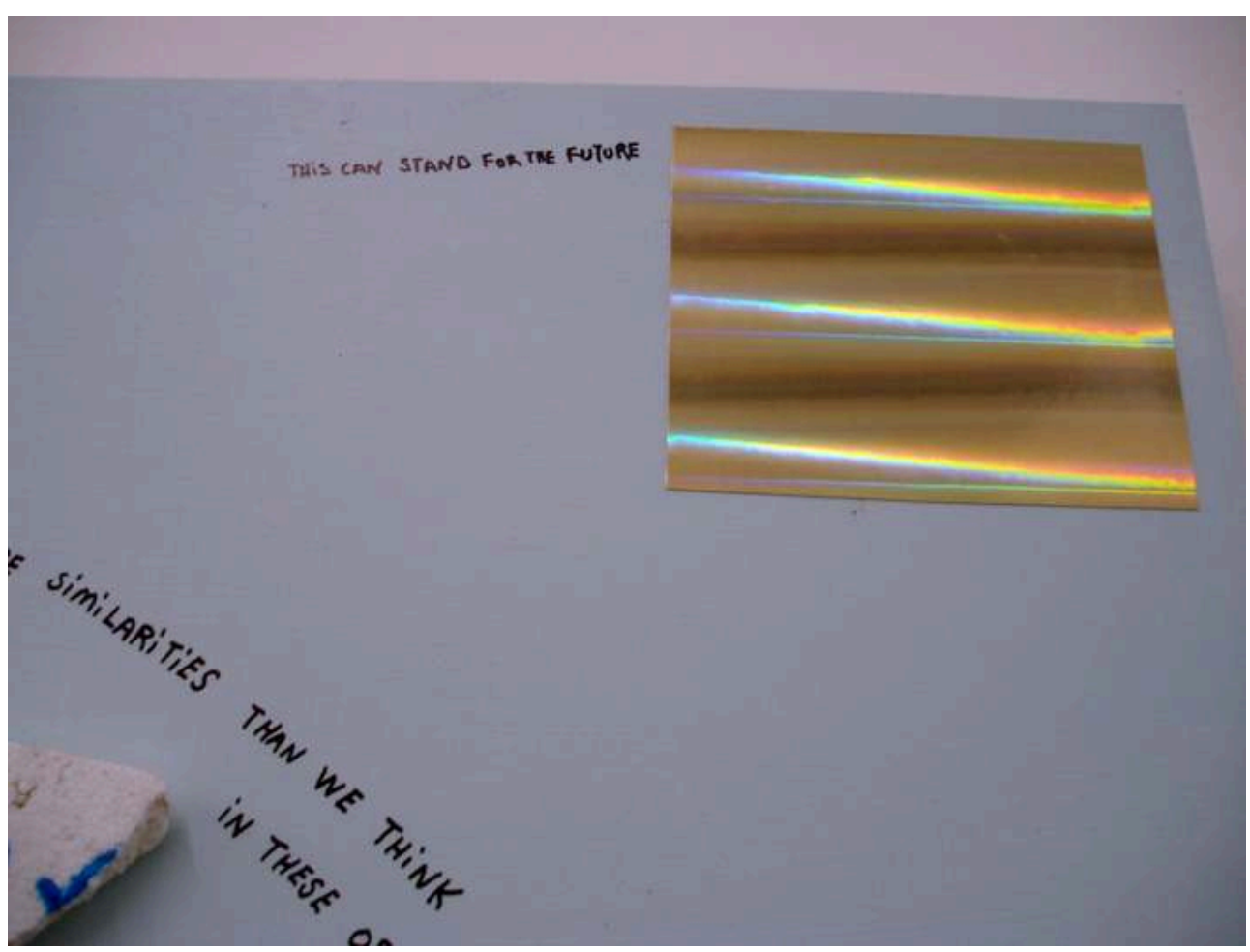

They Almost Fit (2006), détail

Photographie de l'auteure 
Dans le monde de l'art contemporain occidental, rendu plus confus, de nouvelles relations aux objets sont ainsi présentées aux spectateurs de la galerie. Jimmie Durham montre une vie inimaginée des objets, identique à celle du spectateur. Tout d'abord, alors qu'il existe une tendance suivant laquelle «les objets ne sont pas vus en tant qu'eux-mêmes, mais sont abstraits en tant que symboles » et «les choses qui font et nourrissent nos vies (...) sont forcées d'apparaître comme n'étant pas des objets ", il demande que soit réalisée la " possibilité de comprendre ou de connaître la réalité des choses $»^{42}$. Il s'agit d'apprécier l'objet lui-même (matériaux, forme, la relation entre les deux), sa perspective et les mondes dont il peut être le point de vue, en contraste avec celui du spectateur. Snake eyes! (2006) donne un exemple en suggérant plusieurs alternatives: "perhaps these ordinary sea shells can symbolize the current crisis in petroleum » (fig. 12). Les mots dans They almost fit proposent « if this gold leaf can act as a symbol of the past then maybe » et «this can stand for the future... " (figs. 16 et 17). Dans un deuxième temps, nous pouvons considérer les objets comme dictant euxmêmes leur histoire, de leur propre point de vue qu'ils donnent à voir dans la galerie. Dans Snake Eyes! (fig. 18) la vie de ces objets "rejetés» devient digne d'être passionnante. Cette vie a un aspect dramatique mis en évidence par Jimmie Durham, qui semble s'être amusé à monter cette tragédie comme un jeu. Alors, nous les scrutons pour apercevoir qui ils sont par rapport à ce que le texte nous commente. Snake Eyes ! peut, en effet, être approché comme un cartoon, mais qui se raconte lui-même, de deux manières. Les objets le constituant pourraient être d'une part des illustrations de l'histoire qui s'y déroule; nous la suivons en cheminant sur la toile de haut en bas. D'autre part nous pouvons aussi considérer d'abord les objets éparpillés sur la toile comme des personnages d'une histoire qu'ils écrivent, dont ils induisent l'écriture, ou dont ils déclenchent le commentaire rajouté au feutre au-dessous de chacun d'entre eux. La pièce expose un dialogue réciproque entre les différents matériaux, et, à un autre niveau, entre l'artiste et ses matériaux vivants, devant un spectateur lui aussi incorporé avec eux dans la narration. 
Fig.18.

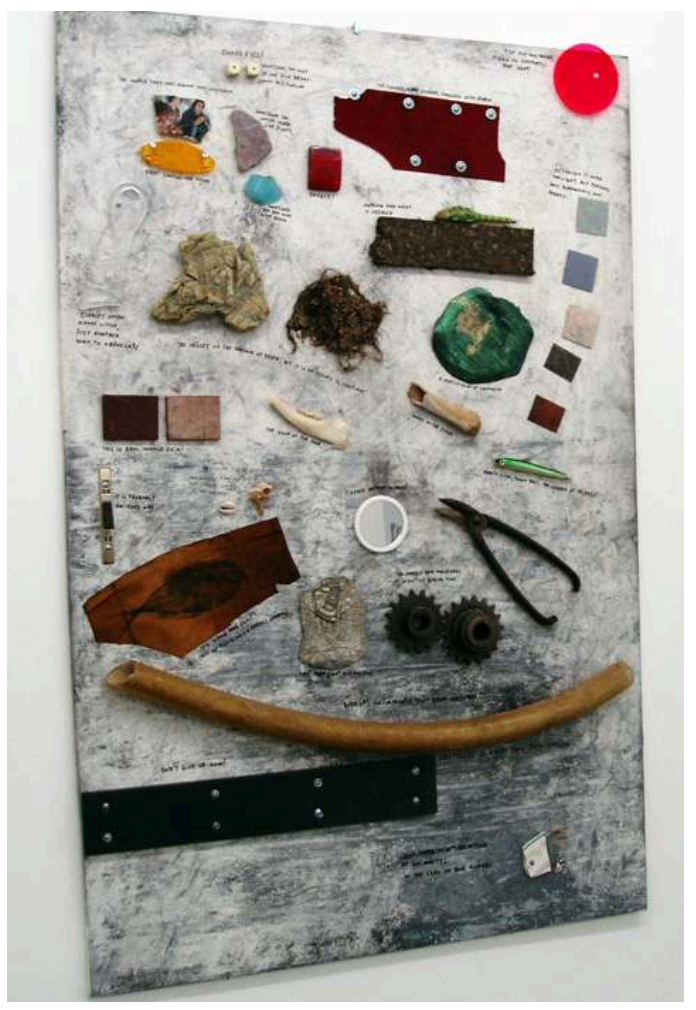

Snake Eyes! (2006)

Photographie de l'auteure

Une multiplicité de communications transparaît dans les œuvres, entre les objets et l'artiste, mais également avec le spectateur. Tout d'abord, l'artiste souhaite engager le spectateur dans une conversation. Un sculpteur ne faisant que « changer les objets ", il souligne que "la question implicite sera toujours 'Pourquoi [l'artiste] a-t-elle [sic] changé cet objet, et qu'est-ce que cela a à voir avec moi ?' $»^{43}$. Puis, l'artiste met en place des relations entre ses œuvres et le spectateur qui les regarde : il établit une voix des œuvres. Pour Jimmie Durham, «le rôle de l'art dans la société est de communiquer ». Il soutient que « faisant partie de la culture humaine, dont le but est la libération humaine, l'art doit avoir pour but l'interprétation sociale critique. Il doit faire partie du dialogue social $»^{44}$. La «réalité des choses» recherchée par Jimmie Durham permettrait au spectateur d'être plus directement engagé avec elles. Les pièces sont de dimensions humaines et exposées à hauteur d'homme, interpellant le spectateur à son niveau : une conversation est ainsi prête à commencer. Alors que la situation dans une galerie d'art tend à l'en empêcher, suivant l'artiste le spectateur doit «danser avec - faire l'expérience de la pièce ». Il voudrait alors que « chaque personne ait une responsabilité de compléter une "sculpture » en entamant une conversation avec elle, une relation $»^{45}$. Une autre stratégie est l'utilisation fréquente de commentaires s'adressant directement au spectateur, par l'emploi de "you». A Mushroom from the Grunewald engage la conversation (fig. 19). Tout d'abord le champignon, étrange chose ronde et plate sur une peinture nacrée, attire l'oeil. Il veut parler et sa parole semble bénéficier de la note au pied du mur indiquée par un astérisque - sorte de bulle de cartoon. Après la description interrompue que nous avons vue, Jimmie Durham (artiste et son double dans l'oeuvre: le signataire du 
commentaire) prend le parti de l'oeuvre. Il interpelle directement le spectateur et lui demande d'assimiler sa position : " yes, it does look a bit strange ; nevertheless. you are also a bit strange, remember only about a month ago when you overheard these friends talking about you? i think you did not hear enough to catch on, luckily, maybe. but anyway $i$ am on your side... if they knew about you (up to a certain point, of course) they would surely have more respect. » Cette interpellation frappante nous fait alors comprendre que le champignon (et son texte) nous est semblable. Nous pouvons le regarder et l'observer avec considération. Les remarques peuvent être plus confondantes envers le spectateur : "You think that the world will demand a complete explanation from you. But the world has forgotten thinking of other things. José Saramago " (fig. 20); cette voix ambiguë pouvant être autant celle de l'auteur ou de l'artiste que celle de l'oeuvre malicieuse. Le spectateur est impliqué dans la pièce Snake Eyes! . Il n'est pas seulement témoin de ce drame, mais il est directement appelé à compatir par une injonction « it could happen to you!», sa propre image est incérée dans la pièce par son reflet dans un miroir : il est invité au même niveau que les objets/ personnages (fig. 14). Dans cette mesure les identifications avec les personnages semblent fonctionner comme devant une fiction. Dans l'oeuvre s'adressant au spectateur sont donc perceptibles les voix de l'artiste (qui produit l'objet et qui commente), et de l'oeuvre elle-même.

Fig. 19.

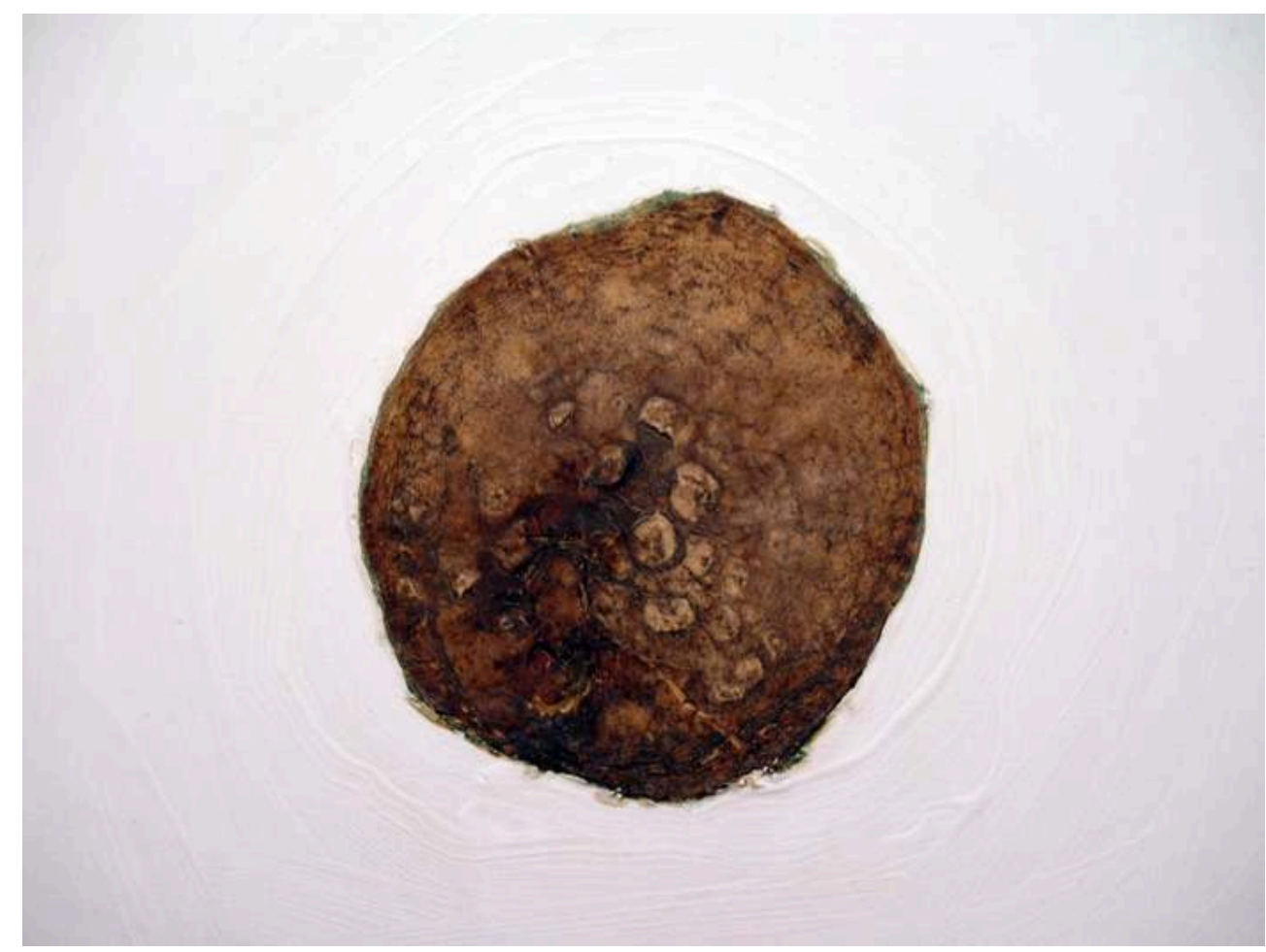

A Mushroom from the Grunewald (2006), détail

Photographie de l'auteure 
Fig.20.

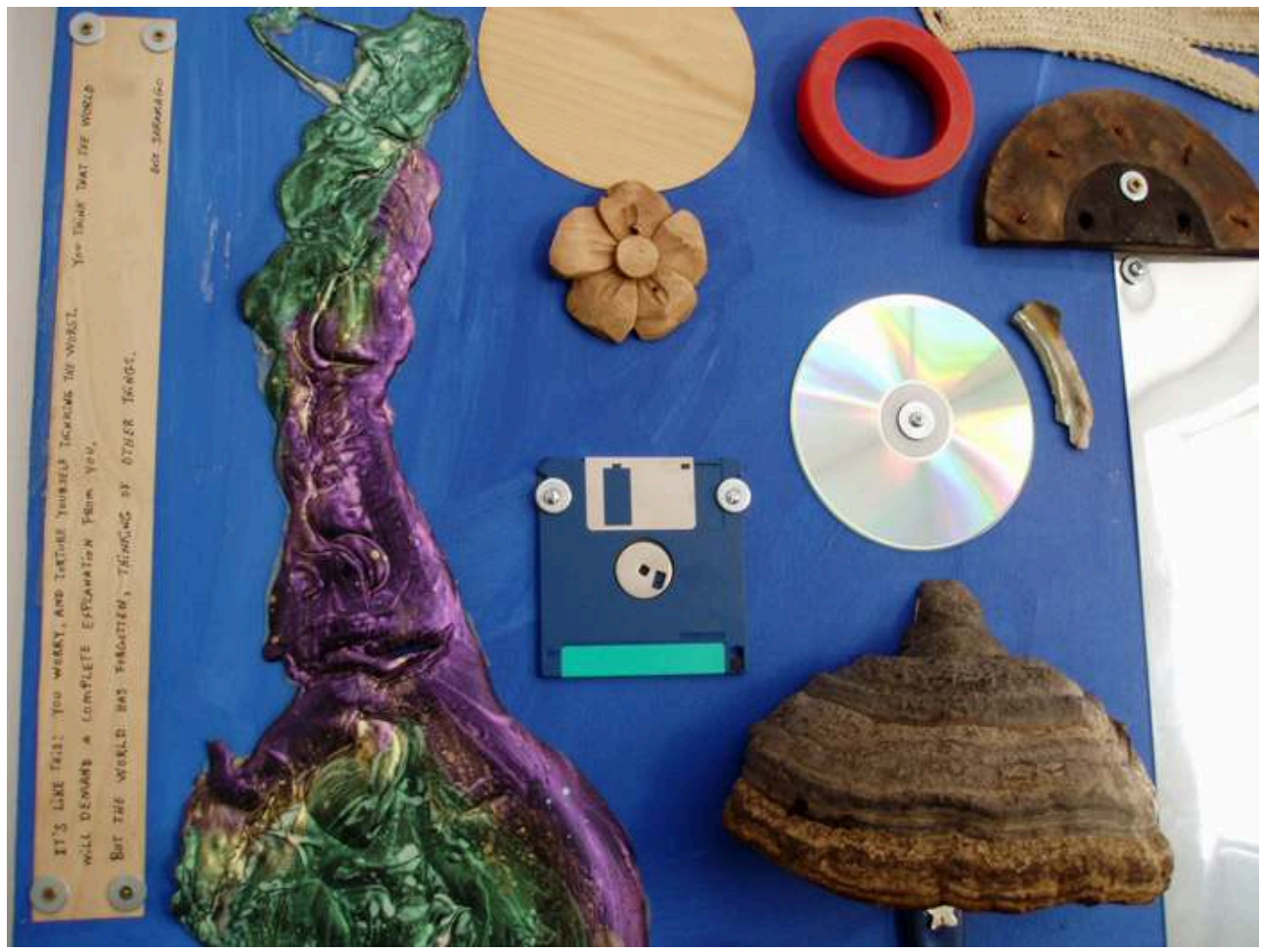

16 red objects and a pecan (2006), détail

Photographie de l'auteure

\section{Dialogues à voix multiples}

Un aspect qui contribue à la capacité de communication et à la vie des objets est leur caractère indéfinissable allié à l'importance de leurs propriétés matérielles. Pour la réalisation du carton de l'exposition, l'artiste commence par vouloir portraiturer un des éléments de la pièce qui donne son nom à l'exposition, et dessine finalement une chose les confondant tous, une chose encore plus indéfinissable (fig. 21). Souvent les éléments constituant les œuvres sont des parties d'autres objets cassés ou incomplets. Ils ne racontent pas leurs vies antérieures de manière évidente, ils nous obligent à faire des inférences, forcent l'imagination à travailler. Ce manque de définitions claires produit un dialogue entre l'objet et le spectateur. Nous pouvons alors considérer les objets comme « vivants » en ce sens. Le pouvoir de communication est renforcé par les stratégies développées par Jimmie Durham pour confondre les catégories occidentales $d^{\prime}$ « art », d'« artiste », d'« indien », etc. Les combinaisons et connexions qu'il effectue égarent le spectateur dans sa perception des œuvres et dans ses concepts pour les comprendre. Nous avons déjà remarqué qu'il doit associer différents cadres pour lire certaines pièces, et que les objets font appel à la possibilité de points de vue différents du sien. L'œuvre de Jimmie Durham est ainsi un objet pour une multiplicité de perspectives différentes, dont nous avons esquissé quelques exemples. Ces perspectives sont comprises dans les différents modes d'approches des objets d'art. D'un côté en tant qu'«art», impliquant une certaine approche, par exemple esthétique ou institutionnalisée. D'un autre côté comme "objets-personnes" ou choses dotées d'intentionnalités, qui sont à considérer suivant leur présence matérielle, leurs qualités 
et leurs relations, et suivant les entités comprises dans ces relations. De plus, ces perspectives sont accumulées dans un même objet. Nous avons vu qu'un objet peut avoir une histoire complexe, subir des transformations et appartenir à différents mondes, être constitué d'intentionnalités et d'aspects qui peuvent être contradictoires - le but de l'artiste Jimmie Durham est en effet de dérouter les spectateurs de ses œuvres.

Fig.21.

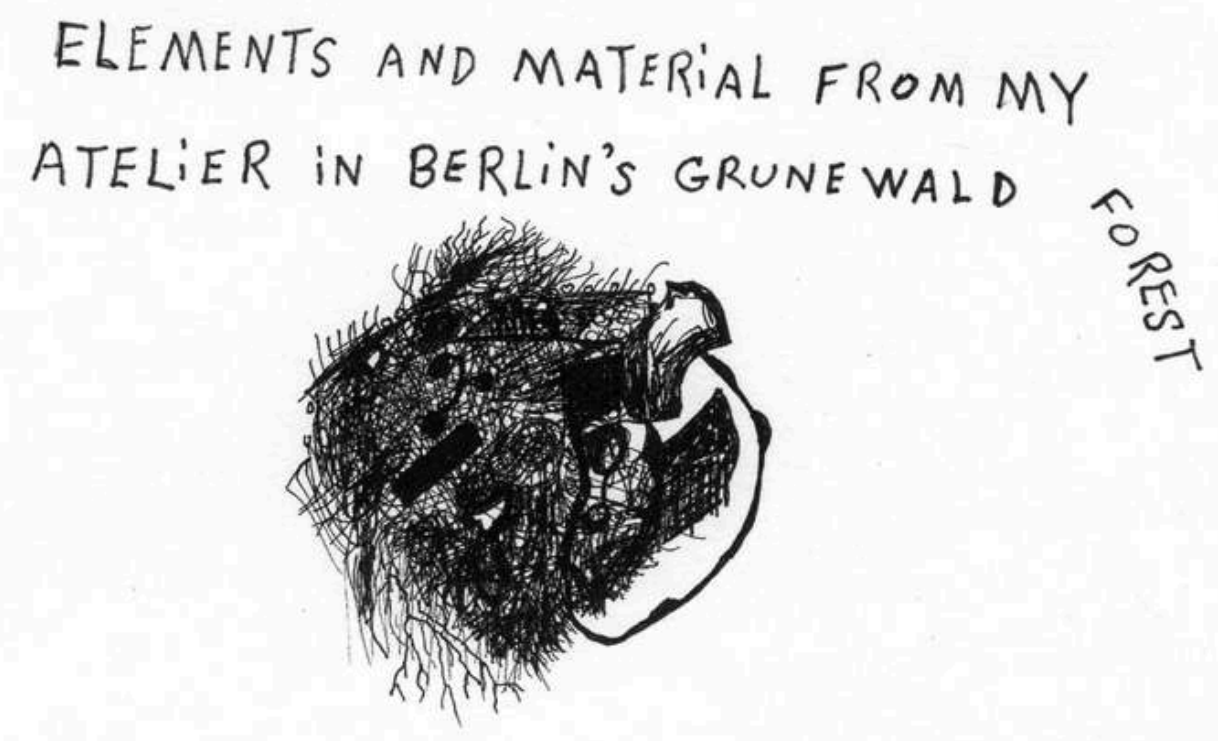

Carton de l'exposition (carte postale) (2006)

Une multiplicité de voix et d'adresses est remarquable, souvent au sein d'une même pièce. Carlo Severi a montré qu'un "énonciateur complexe", "capable de prêter sa voix à différents êtres invisibles ", peut être construit en basant son identité sur " une condensation de connotations contradictoires $»^{46}$. Un tel énonciateur pourrait se situer simultanément dans différents mondes et percevoir les choses selon différentes perspectives : celle de l'artiste et celle de l'objet par exemple, tout en impliquant celle du spectateur. Il faut remarquer de surcroît que ces modes d'approches sont articulés par et autour de l'artiste qui a lui-même une identité complexe comprise dans différents contextes: "bien que [son œuvre] assez naturellement reflète [son] background de certaines manières, souvent il est concerné par la façon dont les blancs s'identifient eux-mêmes et le monde $»^{47}$; il a en effet une profonde connaissance tant de la culture occidentale que de la culture cherokee. Son œuvre est inscrite dans et dirigée vers le monde de l'art contemporain. Jimmie Durham semble appliquer dans le contexte de l'art occidental, en étant de plus situé et engagé localement (les éléments viennent de son studio Berlinois), une mise en place de relations qui demande une considération inhabituelle des pièces par les spectateurs de la galerie d'art. Les éléments utilisés, ayant d'autres connotations dans le contexte occidental (choses trouvées, morceaux, rejets, par exemple), sont alors dotés d'intentionnalités définies par interactions. Un nouveau «message " apparaît ${ }^{48}$. Les objets sont insérés dans des 
nouveaux contextes, dont celui de l'art contemporain, et deviennent des objets d'art par cette action de l'artiste. Les réseaux relationnels compris dans différents mondes que nous avons entrevus établissent les diverses définitions simultanées de l'objet, qui devient une œuvre d'art avec des intentionnalités particulières et mise en scènes de manière "captivante» pour le spectateur. Il est alors actif en déclenchant les questionnements face à la complexité de ses identités accumulées.

\section{Déranger l'imagination - transformer le spectateur}

Les objets peuvent témoigner d'autres mondes à partir de leurs points de vue différents. Ces points de vue sont rendus possibles par le fait que nous pouvons concevoir les objets comme des personnes. Entités intentionnelles, ils contiennent différentes voix qui sont présentes, et pas seulement dans les textes. Les éléments des œuvres de Jimmie Durham valent autant que les textes, leurs matériaux et leurs qualités engagent autant de relations que le langage des textes ${ }^{49}$. En face de l'objet, le spectateur peut percevoir la voix de l'œuvre, celle de l'artiste, et se comprendre luimême objet de ces adresses. Il peut en effet s'identifier aux pièces par une intersubjectivité et en lisant les textes contenant ces voix. Celle-ci est de plus renforcée par les miroirs incérés dans deux des pièces exposées. Une image de sa propre personne est rendue au spectateur en réflexion. L'objet comprend différentes subjectivités, qui sont réciproquement attribuées entre les éléments de leurs différents points de vue, par exemple : «art » et «choses intentionnelles». Le spectateur quant à lui est situé dans la galerie dans le monde de l'art. Afin de tenter une compréhension de l'objet, amorcée par le dialogue réciproque entre ces mondes, imaginons que, confondu, il peut entrevoir la possibilité de changer de point de vue - d'adopter celui qui comprend la vie des objets présents. Il serait alors capable de se voir lui-même, tel qu'avec son point de vue « artistique » il est entré dans la galerie et de réfléchir sur la vérité de ces normes provenant d'une perspective qui conférait aux pièces une définition différente. La stratégie de Jimmie Durham serait alors effective. D'une part des choses rejetées sont devenues des objets d'art, de l'autre, les catégories dominantes (de l'art par exemple) sont ouvertes en montrant la possibilité de différents points de vue sur les choses du monde. Les accumulations d'identités et de points de vue se situant au sein du même objet, plusieurs mondes réagissent réciproquement en parallèle. En opérant une analogie à une autre échelle, nous pourrions associer cette invitation au changement de point de vue sur les choses, à un commentaire sur la situation des peuples dont le point de vue n'est pas pris en compte - celui des colonisés par exemple (ce qui était le sujet évident et plus direct des œuvres américaines de Jimmie Durham). Ces interactions entre perspectives constituent une compréhension plus complexe des objets et de ce à quoi ils se rapportent. Les différentes adresses et multiples voix dans les œuvres de Jimmie Durham permettent au spectateur d'entrer en conversation avec les pièces, et de leur redonner une place dans le monde. En bouleversant les catégories courantes dans ce monde de l'art occidental, l'artiste permet, par l'intermédiaire de ses œuvres, d'entamer une réflexion libre, dynamique et constructrice sur les objets et les choses. 


\section{NOTES}

1. Les détails de cette exposition sont visibles sur http://www.barbarawien.de.

2. "Press release : Jimmie Durham Elements and Materials from my atelier in Berlin's Grunewald Forest. Jimmie Durham's recently released book begins with a description of the materials he used in the action "The Second Particle Wave Theory" in Sunderland (England): English words, wood, stone, river wear. This unusual description of material is typical of Jimmie Durham's sculptures, in which texts and stones play an active roll [sic]. Aided by a stone, cars are crushed, refrigerators demolished, windows smashed, paint tubes squeezed, or as in the afore mentioned work in Sunderland, a boat is sunk with a boulder. The vocabulary used to speak of art and other circumstances is examined through the accompanying texts. His methods are linguistic research and observations of nature and culture. He identifies himself as a 'theoretical biologist' who depicts the behaviors and norms of cohabitation in various communities. Jimmie Durham's work always contains a distinct critique- directed towards capitalist consumer culture, all forms of racism and the arrogant western notions of art which devalue the rest of the art world as 'folklore'. In a speech at the World Social Forum in Alegre (Brazil), he explicitly spoke for a boycott of the Sao Paulo biennial as Brazil's indigenous people do not have equal human rights. In the current exhibition- his second solo show in our gallery - Jimmie Durham will be showing assemblages and sculptures, objects from his studio in Grunewald in Berlin. He has been working in this spacious studio for years, a space which Hitler had built for Arno Breker in 1940. To some extent, the assemblies reflect upon the historically loaded environment and the architecture of the Nazis but they are also descriptions of found objects which Jimmie Durham found on walks though the forest such as giant mushrooms and plastic trash as well as objects which accumulated in his studio; gloves, tools, luminescent papers, bicycle gears, etc. The assemblages are an inventory of every day things. As worthless as the objects appear to be, they are merged into an image, characterized and accompanied by comments. Jimmie Durham, who has lived in Berlin since 1998, will leave the city for Rome in July. Opening : 28 April 2006 Barbara Wien gallery Linienstrasse 158 - Berlin Hours : Di-Fr 14-19, Sa 12-18".

3. «Jimmie Durham, tournage réalisé à Galerie Michel Rein à Paris, entretien avec l'artiste » sur http://www.creativitv.net/artistes/video/durhamv.html, 2004.

4. "I tried to do my best to make an eccentric discourse of art" (Jimmie Durham, "Dirk Snauwaert in converstation with Jimmie Durham" dans Laura Mulvey, Dirk Snauwert, Mark Alice Grant et Jimmie Durham (éd.), Jimmie Durham, Londres, Phaidon Press, 1995, p. 12).

5. Jimmie Durham dans Nemiroff, Diana, Robert Houle, et Charlotte Townsend-Gault, Land, Spirit, Power: First Nations at the National Gallery of Canada. Ottawa, Ontario, National Gallery of Canada, 1992, p. 144-145.

6. Jimmie Durham note également que «We took glass beads, horses, wool ballets, wheat floor for fry-bread, etc, very early and immediately made them identifiably 'Indian' things. We are able to do that because of our cultural integrity and because our societies are dynamic and able to take in new ideas » (Jimmie Durham, "American Indian Culture : Traditionalism and Spiritualism in a Revolutionary Struggle » [1974] dans Jimmie Durham \& Jean Fisher (éd.), A Certain Lack of Coherence: Writings on Art and Cultural Politics, Londres, Kala Press, 1993, p. 9,21).

7. "Painting (...) only need to be integrated into the public life in situations that involve praxis the mutual give-and-take that produces real change. (...) I believe that art should speak to other parts of life and those parts should speak to art" (Jimmie Durham, "Creativity and the Social Process" [1983] dans A Certain Lack of Coherence, op. cit. , p. 69). 
8. "Our perception of the purpose of our art, as we produce it, must be eminently practical. (...) I think the purpose of our art is to help people interpret their world so that they may be better able to change it in positive ways. That must be the purpose of all human activity" (ibidem).

9. La Déclaration a finalement été adoptée le 13 septembre dernier à New York, malgré l'opposition du Canada, de l'Australie, de la Nouvelle-Zélande et des États-Unis.

10. "Constant change - adaptability (...) it is a tradition that our artists have particularly celebrated and have used to move and strengthen our societies (...). We [artists] feel that by participating in whatever modern dialogues are pertinent we are maintaining this tradition" (Jimmie Durham, "Ni' Go Tlunh A Doh Ka" [1986], dans A Certain Lack of Coherence, op. cit. , p. 108).

11. Nous remarquons ici brièvement que l'artiste démontre une identité apparemment paradoxale, qui est construite en établissant un lien logique entre deux définitions qui se trouvent dans des contextes qui tendent à être contradictoires - perspective de l'art contemporain occidental et point de vue 'Cherokee'. Cela lui permet de produire des oeuvres complexes, comme nous le verrons plus loin, et frappant l'imagination du public. Une manière de réaliser ceci a été démontrée par Carlo Severi dans le cas de mouvements messianiques apaches à la fin du XIXème siècle, centrés sur la personne d'un " prophète » à l'identité paradoxale qui relie les deux définitions en une situation où « Être similaire à toi est être moi par opposition à toi, et vice versa» (voir Severi, Carlo, "Capturing imagination : a cultural approach to cognitive complexity", dans Journal of the Royal Anthropological Institute 10, 2004, p. 819-821). Nous ne développons toutefois pas cet aspect dans l'espace du présent texte.

12. Arno Breker (1900-1991), était sculpteur officiel du parti nazi, et a entretenu des rapports personnels avec Adolf Hitler. Ses oeuvres sont emblématiques l'art fascite-moderniste avec des influences maniéristes et expressionnistes. L'on y trouve, souvent dans des dimensions monumentales, des figures imposantes, des poses inspirées pour exprimer la force de la « race aryenne » et du parti, des adaptations de mythes et des représentations de héros allemands. Son oeuvre post-nazisme est exposée dans le musée adjacent à l'atelier dans la forêt de Grünewald.

13. L'objet peut aussi être compris suivant cadre suivant l'hypothèse de l'intégration conceptuelle dans laquelle certains éléments et relations sont projetés à partir d'espaces d'entrée sur un espace intégrant (Mark Turner et Gilles Fauconnier), et considérer que les objets physiques eux-mêmes sont des données entrées dans le processus du «conceptual blending " : Hutchins les appelle « ancres matérielles» (Edwin Hutchins, « Material anchors for conceptual blends » dans Journal of Pragmatics 37, 2005, p. 1560).

14. Alfred Gell, Art and Agency. An anthropological theory, Oxford, Oxford University Press, 1998.

15. "During the cathedral days art had a clear job: it was to illustrate. And it could illustrate through either painting or sculpture. Art served Narrative, we might say. That is probably why so many people still want art to mean something expressible in language, and assume it to be "meaningless" if it does not. Art served Narrative, but artists wanted to serve something else, something like art" (Jimmie Durham "Second thoughts" dans Jimmie Durham , Milan, Charta, 2004, p. 24).

16. Notons qu'ici la galeriste Barbara Wien s'intéresse avant tout aux connections entre art et langage. Elle est aussi libraire et travaille surtout avec des "artistes engagés avec le livre » (entretien avec Barbara Wien, Berlin, 29.06.2006).

17. Jimmie Durham écrit : "In one of my more famous theories about sculpture (famous, that is, among the voice inside my head; it was never been made public except in some bars), I explain that what a sculptor does is change objects. We take a stone or pieces of metal or plastic and rearrange them to make some order, or non-order, that satisfies us in some way. After Duchamp, this re-arranging can often be simply a matter of placing an object in a different way or in a different place than we would normally expect to see it, or by consecrating it with a signature. The amount of matter I the universe stays the same, at least on the level at which the sculptor may use matter; and all of the possible shapes already exist, at least in a Jungian or Platonic 
sense; so that the 'creation' is not a category of concern for the sculptor" (Jimmie Durham, "Creativity and the Social Process", op. cit., p. 69-70).

18. Jimmie Durham dans Sarah J. Rogers. Will/Power. Exhibition Catalogue, Columbia, Ohio State University, 1993, p. 12. Il est intéressant de remarquer que "[Durham's] refusal of the moment of artistic creation as a zero point at which a new object comes into being from nowhere, condenses with a rejection of the moment when America was created, as a zero date in the history of the continent (Laura Mulvey, « Survey : changing objects, preserving time», dans Jimmie Durham , op. cit. , p. 52).

19. Jimmie Durham, Note explicative pour «Manhattan festival of the dead » dans l'exposition Ritual and Rhythm, New York, Kenkeleba Gallery,1982, p. 3.

20. Jimmie Durham dans Land Spirit Power, op. cit. , p. 145.

21. "When I was a child I started making my own toys at an early age. The toys involved a very complex fantasy life, and any objects I could handle was under the constant threat of being transformed into playing a role in that private world.(...) I made my own society in which I had an important part. Something in my make-up caused me to build that society with material objects which I would change one way or another; I just had a 'bent' towards relating to objects and shapes. I suspect this is the case history of many sculptors (...). But as we grow up we are expected to translate this natural tendency to be a critical subject in the work, coupled with whatever are our natural talents, into action in the real world." (Jimmie Durham, "Creativity and the Social Process", op. cit., p. 72).

22. Jimmie Durham dans Land Spirit Power, op. cit, p. 145.

23. Ibidem.

24. Jimmie Durham, «American Indian Culture », op. cit., p. 16-17.

25. Jimmie Durham dans Land Spirit Power , op. cit., p. 146.

26. La pièce Voss Strasse (2004/5), rajoutée à l'exposition, raconte une histoire: comment Jimmie Durham a trouvé deux objets, présentés dans l'oeuvre : les mots suivant concluent le texte qui y est accroché "i had nothing but questions, so i took the two pieces back to my studio". 27. Entretien avec Joen P-Vedel, alors assistant de Jimmie Durham, Berlin, 30.04.2006.

28. * THIS IS A DRIED MUSHROOM, THAT IS TO SAY, A FUNGUS, FROM THE GRUNEWALD. GOEDELS NOBELS, JOEN VEDEL AND I FOUND IT. WE BELIVE BELIEVE IT TO BE A 'DESTROYINg ANGEL' (AMANITA VIROSA) BUT IT MIGHT BE INSTEAD THE 'DECEIVER' (LACCARIA LACCATA) OR POSSIBLY ' LEUCOAP LEUCOAGARICUS PUDICUS, OR EVEN CYSTOLEPIATA ADULTERINA; MAYBE IT IS THE 'EARTHSTAR' (GEASTRUM FORNICATUM) WHICH IS INEDIBLE BECAUSE OF THE TEXTURE. WE CONSULTED THE REFERENCE BOOKS AND ESTABLISHED THAT IT IS NOT 'STINKHORN' (PHALLUS IMPUDICUS). AFTER DRYING IT I SOAKED IT IN A MIXTURE OF WHITE GLUE, WATER AND ALCOHOL (DID YOU KNOW THAT WHITE GLUE IS MADE FROM COWS' MILK?) IT IS NOW COMPLETELY PRESERVED, BECAUSE AFTER IT DRIED A SECOND TIME I APPLIED ACRYLIC GEL; YES, IT DOES LOOK A BIT STRANGE; NEVERTHELESS. YOU ARE ALSO A BIT STRANGE, REMEMBER ONLY ABOUT a MONTH AGO WHEN YOU OVERHEARD THESE FRIENDS TALKING ABOUT YOU? I THINK YOU DID NOT HEAR ENOUGH TO CATCH ON, LUCKILY, MAYBE. BUT ANYWAY I AM ON YOUR SIDE... IF THEY KNEW ABOUT YOU (UP TO A CERTAIN POINT, OF COURSE) THEY WOULD SURELY HAVE MORE RESPECT. PLEASE BE ASSURED ('ASSURRED'!'ASURRED'!) THAT THIS DESTROYING ANGEL OR DECEIVER IS SUITABLE FOR HOME USE AS WELL AS FOR MUSEUM. Jimmie Durham, BERLIN, SPRING OF 2006

29. Philippe Descola, La Nature Domestique : Symbolisme et Praxis dans l'Écologie des Achuar, Paris, Éditions de la MSH, 1986, p. 120.

30. Eduardo Viveiros de Castro, "Perspectival anthropology and the method of controlled equivocation" dans Tipití Journal of the Society for the Anthropology of Lowland South America 2:2, 2004 (ma traduction).

31. Ibidem. 
32. Ibidem.

33. Voir par exemple Severi, Carlo \& Michael Houseman, Naven ou le Donner à voir. Essai d'interprétation de l'action rituelle, Paris, MSH, 1994.

34. Conversation avec Jimmie Durham, Berlin, 12.07.2006.

35. "When we work on paper we cannot forget the paper-making process, the fact of the trees on our land. (...) What semiological languages can we expose? Languages speak through objects. A whisky bottle has as valid an artistic use as any other object. Of course, one listens to the conversations of the bottle when considering aesthetic purposes. It would be something like a sin to trick the bottle into lying: a matter of intercepting the universal dialogue so necessary to our survival. One can use the bottle to play tricks, or to tell jokes, or to make any unexpected combinations, but we would not force it into false positions" (Jimmie Durham, "Ni' Go Tlunh A Doh Ka", op. cit., p.110).

36. "We don't accept ideas of 'development' or 'economic growth' unless we can clearly see the long-range and short-range benefits they will provide to human beings. (...) We might also call this value the 'spirit of things'. The concept unables us to choose. We accept telephones. We do not accept DDT or napalm" (Jimmie Durham, "American Indian Culture : Traditionalism and Spiritualism in a Revolutionary Struggle », op. cit, p. 17).

37. "I would like to make art each individual thing there is, there would not be a time when you had to decide to keep it or throw it away. And, it seems to me, one can do that sort of nondictatorial thing by making things which don't have to do with craftwork at all, just intellectually joined our normal physical world" (Jimmie Durham, "Dirk Snauwaert in converstation with Jimmie Durham" op. cit. , p. 25).

38. Le livre de Jimmie Durham, The Second Particle Wave Theory The Second Particle Wave Theory: As Performed on Banks of the River Wear, a Stone's Throw from S'Underland and the Durham Cathedral, Banff Centre Press, 2005, ouvre sur une liste des «matériaux utilisés » dans sa performance du même nom en Angleterre : « English words ", « Wood ", "Stone », « The Wear river ».

39. Jimmie Durham «In the Joyeria of the Zanahorias» dans Semi-specific objects and almostrelated words, Düren, Leopold-Hoesch Museum und Peill-Stiftung, 2003, p. 28.

40. "I do not like language (but it does not follow that I advocate its disuse or misuse - just the contrary), but I love words. (Please forgive this digression, for some reason we speak as though there were a connection between words and objects. One of the interesting properties of both words and objects is that they repel each other. I often like to combine them in art works by nailing or gluing them together because of the vibration this causes)" (Jimmie Durham, «Second thoughts ", op. cit., p. 22).

41. Jimmie Durham dans Land Spirit Power, op. cit., p. 146.

42. Jimmie Durham, «American Indian Culture », op. cit., p. 18-19.

43. Jimmie Durham explique : "what a sculptor does is change objects (...) we could substitute, 'he created a new piece' for 'she changed another object'. The implied question would always be, "Why did she change that object, and what does that have to do with me?" (Jimmie Durham, "Creativity and the Social Process", op. cit., p. 69-70).

44. "The role of art and of artists, then, must be seen in that context. As a part of human culture, which has the purpose of human liberation, art must have the purpose of critical social interpretation. It must be part of the social dialogue, and not separated by theories of aesthetics or by middle-class edification" (ibidem).

45. "My idea is that, like in my own culture, each person would have a responsibility to complete a «sculpture » by beginning a conversation with it, a relationship » (Jimmie Durham, Note explicative pour « Manhattan festival of the dead », op. cit., 1982, p. 3,4).

46. Severi, Carlo, "Memory, Reflexivity and Belief : Reflections on the Ritual Use of Language", dans Social Anthropology 10:1, 2002, p. 33-34, 36. 
47. Jimmie Durham dans Richard Shiff, "The Necessity of Jimmie Durham's Jokes", dans Art Journal 51:3, 1992, p. 75.

48. Voir Severi, Carlo, "Capturing imagination : A cultural approach to cognitive complexity", op.cit., 2004, p. 836.

49. Mentionnons en outre cet aspect de la langue cherokee que Jimmie Durham a souligné : « les noms [cherokee] sont ne sont pas des noms du tout, mais des descriptions d'action, de fonction ou de forme (ou les trois ensemble). Nous pouvons considérer [le cherokee] comme une langue qui parle de fonctions et de relations ", positionnant et engageant ainsi celui qui parle avec les choses de son entourage (Jimmie Durham, « Gado usdi hia? » dans Rendez(-) Vous Objects, Gent, Museum Van Hedendaagse Kunst, 1993, p. 7).

\section{RÉSUMÉS}

Les œuvres de Jimmie Durham confondent systématiquement ceux qui les regardent. Elles possèdent certaines qualités étranges qui transforment des choses communes, bancales, cassées ou rejetées en déclencheurs d'investigations. Dans une galerie d'art contemporain, ce sont des objets d'art. Jimmie Durham établit, modifie et superpose dans ses œuvres les identités d'objets éclectiques. À l'occasion d'une exposition qui eut lieu à Berlin en 2006, nous envisageons comment, par imputations de subjectivités, ils peuvent acquérir des qualités de "personnes » et entamer des conversations, « dialoguer » avec les spectateurs.

\section{INDEX}

Mots-clés : objets trouvés, œuvre d'art, chose, matériau, galerie

\section{AUTEUR}

\section{SOPHIE MOIROUX}

Sophie Moiroux est actuellement doctorante en anthropologie sociale à l'EHESS sous la direction de Carlo Severi (anthropologie de l'art). Elle étudie l'art contemporain, en particulier l'œuvre de Jimmie Durham, et réalise également une recherche sur l'art d'artistes indigènes du Brésil. sophie_m_m@yahoo.fr 